\title{
Relative controllability of linear difference equations
}

\author{
Guilherme Mazanti*
}

February 20, 2017

\begin{abstract}
In this paper, we study the relative controllability of linear difference equations with multiple delays in the state by using a suitable formula for the solutions of such systems in terms of their initial conditions, their control inputs, and some matrix-valued coefficients obtained recursively from the matrices defining the system. Thanks to such formula, we characterize relative controllability in time $T$ in terms of an algebraic property of the matrix-valued coefficients, which reduces to the usual Kalman controllability criterion in the case of a single delay. Relative controllability is studied for solutions in the set of all functions and in the function spaces $L^{p}$ and $\mathrm{C}^{k}$. We also compare the relative controllability of the system for different delays in terms of their rational dependence structure, proving that relative controllability for some delays implies relative controllability for all delays that are "less rationally dependent" than the original ones, in a sense that we make precise. Finally, we provide an upper bound on the minimal controllability time for a system depending only on its dimension and on its largest delay.
\end{abstract}

Notations In this paper, we denote by $\mathbb{N}$ and $\mathbb{N}^{*}$ the sets of nonnegative and positive integers, respectively. For $a, b \in \mathbb{R}$, we write the set of all integers between $a$ and $b$ as $\llbracket a, b \rrbracket=[a, b] \cap \mathbb{Z}$, with the convention that $[a, b]=\emptyset$ if $a>b$. The cardinality of a set $\mathcal{N}$ is denoted by $\# \mathcal{N}$. For $\xi \in \mathbb{R}^{N}$, we use $\xi_{\min }$ and $\xi_{\max }$ to denote the smallest and the largest components of $\xi$, respectively. For $\xi \in \mathbb{R}$, the symbol $\lfloor\xi\rfloor$ is used to the denote the integer part of $\xi$, i.e., the unique integer such that $\xi-1<\lfloor\xi\rfloor \leq \xi$.

The set of $d \times m$ matrices with coefficients in $K \subset \mathbb{C}$ is denoted by $\mathcal{M}_{d, m}(K)$, or simply by $\mathcal{M}_{d}(K)$ when $m=d$. The identity matrix in $\mathcal{M}_{d}(\mathbb{C})$ is denoted by $\operatorname{Id}_{d}$ and the zero matrix in $\mathcal{M}_{d, m}(\mathbb{C})$ is denoted by $0_{d, m}$, or simply by 0 when its dimensions are clear from the context. We use $e_{1}, \ldots, e_{d}$ to denote the canonical basis of $\mathbb{C}^{d}$. For $p \in[1,+\infty],|\cdot|_{p}$ indicates both the $\ell^{p}$-norm in $\mathbb{C}^{d}$ and the corresponding induced matrix norm in $\mathcal{M}_{d, m}(\mathbb{C})$. The range of a matrix $M \in \mathcal{M}_{d, m}(\mathbb{C})$ is denoted by $\operatorname{Ran} M$, and $\operatorname{rk} M$ denotes the dimension of $\operatorname{Ran} M$.

This article was prepared while the author was with CMAP \& Inria, team GECO, École Polytechnique, CNRS, Université Paris-Saclay, 91128 Palaiseau Cedex, France. This research was partially supported by the iCODE Institute, research project of the IDEX Paris-Saclay, and by the Hadamard Mathematics LabEx (LMH) through the grant number ANR-11-LABX-0056-LMH in the "Programme des Investissements d'Avenir".

2010 Mathematics Subject Classification. 39A06, 93B05, 93C23, 93B25.

Keywords. Relative controllability, difference equations, delays, explicit solution, rational dependence, minimal controllability time.

*Laboratoire de Mathématiques d'Orsay, Univ. Paris-Sud, CNRS, Université Paris-Saclay, 91405 Orsay, France. (guilherme.mazanti@math.u-psud.fr, http://www . math.u-psud.fr/ mazanti). 


\section{Introduction}

This paper characterizes the relative controllability of the controlled difference equation

$$
\Sigma(A, B, \Lambda): \quad x(t)=\sum_{j=1}^{N} A_{j} x\left(t-\Lambda_{j}\right)+B u(t),
$$

where $x(t) \in \mathbb{C}^{d}$ is the state, $u(t) \in \mathbb{C}^{m}$ is the control input, $N, d, m \in \mathbb{N}^{*}, \Lambda=\left(\Lambda_{1}, \ldots, \Lambda_{N}\right) \in(0,+\infty)^{N}$ is the vector of positive delays, $A=\left(A_{1}, \ldots, A_{N}\right) \in \mathcal{M}_{d}(\mathbb{C})^{N}$ is a $N$-tuple of $d \times d$ complex-valued matrices, and $B \in \mathcal{M}_{d, m}(\mathbb{C})$ is a $d \times m$ complex-valued matrix.

An important motivation for the study of (1.1) is that several hyperbolic PDEs can be transformed into such system thanks to classical transformations based mainly on the method of characteristics $[6,7,14,21,32]$. In particular, stability criteria for transport and wave equations on networks have been obtained in [4] through the stability analysis of (1.1) with no control input, and a similar method has been used in [7] to characterize the stability of nonlinear hyperbolic systems with respect to the $\mathrm{e}^{1}$ and $W^{1, p}$ norms.

Another motivation comes from the study of more general neutral functional differential equations of the form

$$
\frac{d}{d t}\left(x(t)-\sum_{j=1}^{N} A_{j} x\left(t-\Lambda_{j}\right)\right)=f\left(x_{t}\right)+B u(t),
$$

where $x_{t}:[-r, 0] \rightarrow \mathbb{C}^{d}$ is given by $x_{t}(s)=x(t+s), r \geq \max _{j \in \llbracket 1, N \rrbracket} \Lambda_{j}$, and $f$ is some function defined on a certain space (typically $\mathfrak{C}^{k}\left([-r, 0], \mathbb{C}^{d}\right)$ or $\left.W^{k, p}\left((-r, 0), \mathbb{C}^{d}\right)\right)[8,9,15,24]$, [16, Section 9.7]. It has been proved in [18] that, under no control, there is a deep link between the dynamic properties of (1.1) and (1.2), due to the fact that the essential spectra of the associated semigroups coincide. Such link has been exploited, for instance, in [17] to obtain criteria for the stabilizability of (1.1) and (1.2) under linear state feedbacks. Other works have also considered control and stabilization properties for (1.2), such as [25,26, 28,30].

The stability analysis of (1.1) with no control input has a long history [1, 8-10, 15, 18, 22] (see also [16, Chapter 9] and references therein). In particular, it has been shown that the stability of (1.1) is not preserved under perturbations of the delays $[8,16,18,22]$, and that the rational dependence of the delays plays an important role in the stability analysis $[1,4,18,23,31]$. Such interplay between rational dependence of the delays and properties of (1.1) is also present when one considers relative controllability, as we show in Section 4.

Concerning the controllability problem, due to the infinite-dimensional nature of the dynamics of neutral functional differential equations and difference equations, several different notions of controllability can be used, such as exact, approximate, spectral, or relative controllability [5,30]. Relative controllability has been originally introduced in the study of control systems with delays in the control input $[5,20,27]$, but this notion has later been extended and used to study also systems with delays in the state [13,29] and in more general frameworks, such as for stochastic control systems [19] or fractional integro-differential systems [2]. The main idea of relative controllability is that, instead of controlling the state $x_{t}:[-r, 0] \rightarrow \mathbb{C}^{d}$ of $(1.1)$, defined by $x_{t}(s)=x(t+s)$, in a certain function space such as $\mathcal{C}^{k}\left([-r, 0], \mathbb{C}^{d}\right)$ or $L^{p}\left((-r, 0), \mathbb{C}^{d}\right)$, where $r \geq \max _{j \in \llbracket 1, N \rrbracket} \Lambda_{j}$, one controls only the final state $x(t)=x_{t}(0)$. We defer the precise definition of relative controllability used in this paper to Definition 3.4, after having proved in Theorems 3.1 and 3.2 criteria for several equivalent or closely related notions of relative controllability.

The relative controllability of systems related to (1.1) has been addressed in $[11,13,29]$, where, motivated by the analysis of the relative controllability of the continuous-time delayed control system $\dot{x}(t)=A_{0} x(t-\tau)+B_{0} u(t)$, the authors consider a discrete-time system under the form

$$
\Delta x(t)=A x(t-k)+B u(t), \quad t \in \mathbb{N},
$$


where $\Delta x(t)=x(t+1)-x(t)$ and $k \in \mathbb{N}^{*}$. Such system corresponds to an explicit Euler discretization of the continuous-time system $\dot{x}(t)=A_{0} x(t-\tau)+B_{0} u(t)$ with time step $h=\frac{\tau}{k}$ and $A=h A_{0}, B=$ $h B_{0}$. Using an explicit representation of solutions based on discrete delayed matrix exponentials, the authors characterize the relative controllability of (1.3) and the minimal controllability time, and provide expressions for the control input steering the system from a prescribed initial condition to a desired final state. A comparison between the results of this paper and those from [13] is provided in Example 5.6.

In this paper, the relative controllability of (1.1) is analyzed through a suitable representation formula for its solutions, describing a solution in time $t$ in terms of its initial condition, the control input, and some matrix-valued coefficients computed recursively (see Proposition 2.7). Such coefficients generalize the discrete delayed matrix exponentials introduced in [12] for (1.3) to the case of several delays and matrices. A similar formula has been used in [3] to analyze the stability of a system of transport equations on a network under intermittent damping and in [4] to obtain stability criteria for (1.1) under no control and with time-varying matrices $A_{j}$, which in particular provide generalizations of classical stability results for difference equations such as the Hale-Silkowski criterion from [31] (cf. also [1], [16, Section 9.6]).

The plan of the paper is as follows. After some general discussion on the well-posedness of (1.1) and the derivation of the explicit representation formula for its solutions in Section 2, we characterize relative controllability for some fixed final time $T>0$ in Section 3 in the set of all functions and in the function spaces $L^{p}$ and $\mathcal{C}^{k}$. For given $A=\left(A_{1}, \ldots, A_{N}\right) \in \mathcal{M}_{d}(\mathbb{C})^{N}$ and $B \in \mathcal{M}_{d, m}(\mathbb{C})$, Section 4 compares the relative controllability of (1.1) for different delays $\Lambda_{1}, \ldots, \Lambda_{N}$ and $L_{1}, \ldots, L_{N}$ in terms of their rational dependence structure. Finally, Section 5 provides a uniform upper bound on the minimal time for the relative controllability of (1.1).

Notice that all the results in this paper also hold, with the same proofs, if one assumes $A=$ $\left(A_{1}, \ldots, A_{N}\right) \in \mathcal{M}_{d}(\mathbb{R})^{N}$ and $B \in \mathcal{M}_{d, m}(\mathbb{R})$ with the state $x(t) \in \mathbb{R}^{d}$ and the control $u(t) \in \mathbb{R}^{m}$. We choose complex-valued matrices, states, and controls for (1.1) in this paper following the approach of [4], which is mainly motivated by the fact that classical spectral conditions for difference equations are more naturally written down in such framework.

\section{Well-posedness and explicit representation of solutions}

This sections establishes the well-posedness of (1.1) and provides an explicit representation formula for its solutions. The proofs of the main results of this section, Propositions 2.2 and 2.7, are very similar to the ones given in [4] for the corresponding uncontrolled system, and for such reason are omitted here. We start by providing the definition of solution used in this paper.

Definition 2.1. Let $A=\left(A_{1}, \ldots, A_{N}\right) \in \mathcal{M}_{d}(\mathbb{C})^{N}, B \in \mathcal{M}_{d, m}(\mathbb{C}), \Lambda=\left(\Lambda_{1}, \ldots, \Lambda_{N}\right) \in(0,+\infty)^{N}, T>0$, $x_{0}:\left[-\Lambda_{\max }, 0\right) \rightarrow \mathbb{C}^{d}$, and $u:[0, T] \rightarrow \mathbb{C}^{m}$. We say that $x:\left[-\Lambda_{\max }, T\right] \rightarrow \mathbb{C}^{d}$ is a solution of $\Sigma(A, B, \Lambda)$ with initial condition $x_{0}$ and control $u$ if it satisfies (1.1) for every $t \in[0, T]$ and $x(t)=x_{0}(t)$ for $t \in\left[-\Lambda_{\max }, 0\right)$.

For $t \in[0, T]$ and $x:\left[-\Lambda_{\max }, T\right] \rightarrow \mathbb{C}^{d}$ a solution of $\Sigma(A, B, \Lambda)$, we define $x_{t}:\left[-\Lambda_{\max }, 0\right) \rightarrow \mathbb{C}^{d}$ by $x_{t}=\left.x(t+\cdot)\right|_{\left[-\Lambda_{\max }, 0\right)}$.

Notice that this definition of solution contains no regularity assumptions on $x_{0}, u$, or $x$. Nonetheless, this weak framework is enough to guarantee existence and uniqueness of solutions, as stated in the next proposition, whose proof is very similar to that of [4, Proposition 3.2].

Proposition 2.2. Let $A=\left(A_{1}, \ldots, A_{N}\right) \in \mathcal{M}_{d}(\mathbb{C})^{N}, B \in \mathcal{M}_{d, m}(\mathbb{C}), \Lambda=\left(\Lambda_{1}, \ldots, \Lambda_{N}\right) \in(0,+\infty)^{N}, T>$ $0, x_{0}:\left[-\Lambda_{\max }, 0\right) \rightarrow \mathbb{C}^{d}$, and $u:[0, T] \rightarrow \mathbb{C}^{m}$. Then $\Sigma(A, B, \Lambda)$ admits a unique solution $x:\left[-\Lambda_{\max }, T\right]$ $\rightarrow \mathbb{C}^{d}$ with initial condition $x_{0}$ and control $u$. 
Remark 2.3. Let $T>0$. If $x_{0}, \widetilde{x}_{0}:\left[-\Lambda_{\max }, 0\right) \rightarrow \mathbb{C}^{d}$ and $u, \widetilde{u}:[0, T] \rightarrow \mathbb{C}^{m}$ are such that $x_{0}=\widetilde{x}_{0}$ and $u=\widetilde{u}$ almost everywhere on their respective domains, then the solutions $x, \tilde{x}:\left[-\Lambda_{\max }, T\right] \rightarrow \mathbb{C}^{d}$ of $\Sigma(A, B, \Lambda)$ associated respectively with $x_{0}, u$, and $\widetilde{x}_{0}, \widetilde{u}$, satisfy $x=\widetilde{x}$ almost everywhere on $\left[-\Lambda_{\max }, T\right]$. In particular, one still obtains existence and uniqueness of solutions of $\Sigma(A, B, \Lambda)$ (in the sense of functions defined almost everywhere) for initial conditions in $L^{p}\left(\left(-\Lambda_{\max }, 0\right), \mathbb{C}^{d}\right)$ and controls in $L^{p}\left((0, T), \mathbb{C}^{m}\right)$ for some $p \in[1,+\infty]$, any such solution $x$ satisfies $x \in L^{p}\left(\left(-\Lambda_{\max }, T\right), \mathbb{C}^{d}\right)$, and hence $x_{t} \in L^{p}\left(\left(-\Lambda_{\max }, 0\right), \mathbb{C}^{d}\right)$ for every $t \in[0, T]$.

Remark 2.4. If $x_{0} \in \mathcal{C}^{k}\left(\left[-\Lambda_{\max }, 0\right), \mathbb{C}^{d}\right)$ and $u \in \mathcal{C}^{k}\left([0, T], \mathbb{C}^{m}\right)$ for some $k \in \mathbb{N}$, then the corresponding solution $x$ of $\Sigma(A, B, \Lambda)$ belongs to $\mathcal{C}^{k}\left(\left[-\Lambda_{\max }, T\right], \mathbb{C}^{d}\right)$ if and only if

$$
\lim _{t \rightarrow 0} x_{0}^{(r)}(t)=\sum_{j=1}^{N} A_{j} x_{0}^{(r)}\left(-\Lambda_{j}\right)+B u^{(r)}(0), \quad \forall r \in \llbracket 0, k \rrbracket,
$$

where $x_{0}^{(r)}$ and $u^{(r)}$ denote the $r$-th derivatives of $x_{0}$ and $u$, respectively.

Due to the compatibility condition (2.1) required for obtaining solutions $x$ in the space $e^{k}\left(\left[-\Lambda_{\max }\right.\right.$, $T], \mathbb{C}^{d}$ ), we find it useful to introduce the following definition.

Definition 2.5. Let $A=\left(A_{1}, \ldots, A_{N}\right) \in \mathcal{M}_{d}(\mathbb{C})^{N}, B \in \mathcal{M}_{d, m}(\mathbb{C}), \Lambda=\left(\Lambda_{1}, \ldots, \Lambda_{N}\right) \in(0,+\infty)^{N}, x_{0}$ : $\left[-\Lambda_{\max }, 0\right) \rightarrow \mathbb{C}^{d}$, and $k \in \mathbb{N}$. We say that $x_{0}$ is $\mathcal{C}^{k}$-admissible for system $\Sigma(A, B, \Lambda)$ if $x_{0} \in \mathrm{C}^{k}\left(\left[-\Lambda_{\max }\right.\right.$, $\left.0), \mathbb{C}^{d}\right)$ and, for every $r \in \llbracket 0, k \rrbracket, \lim _{t \rightarrow 0} x_{0}^{(r)}(t)$ exists and

$$
\lim _{t \rightarrow 0} x_{0}^{(r)}(t)-\sum_{j=1}^{N} A_{j} x_{0}^{(r)}\left(-\Lambda_{j}\right) \in \operatorname{Ran} B .
$$

In order to provide an explicit representation for the solutions of $\Sigma(A, B, \Lambda)$, we first provide a recursive definition of the matrix coefficients $\Xi_{\mathbf{n}}$ appearing in such representation.

Definition 2.6. For $A=\left(A_{1}, \ldots, A_{N}\right) \in \mathcal{M}_{d}(\mathbb{C})^{N}$ and $\mathbf{n} \in \mathbb{Z}^{N}$, we define the matrix $\Xi_{\mathbf{n}} \in \mathcal{M}_{d}(\mathbb{C})$ inductively by

$$
\Xi_{\mathbf{n}}= \begin{cases}0, & \text { if } \mathbf{n} \in \mathbb{Z}^{N} \backslash \mathbb{N}^{N}, \\ \operatorname{Id}_{d}, & \text { if } \mathbf{n}=0, \\ \sum_{k=1}^{N} A_{k} \Xi_{\mathbf{n}-e_{k}}, & \text { if } \mathbf{n} \in \mathbb{N}^{N} \backslash\{0\} .\end{cases}
$$

We now provide an explicit representation for the solutions of $\Sigma(A, B, \Lambda)$, which is a generalization of [4, Lemma 3.13] to the case of the controlled difference equation (1.1).

Proposition 2.7. Let $A=\left(A_{1}, \ldots, A_{N}\right) \in \mathcal{M}_{d}(\mathbb{C})^{N}, B \in \mathcal{M}_{d, m}(\mathbb{C}), \Lambda=\left(\Lambda_{1}, \ldots, \Lambda_{N}\right) \in(0,+\infty)^{N}, T>$ $0, x_{0}:\left[-\Lambda_{\max }, 0\right) \rightarrow \mathbb{C}^{d}$, and $u:[0, T] \rightarrow \mathbb{C}^{m}$. The corresponding solution $x:\left[-\Lambda_{\max }, T\right] \rightarrow \mathbb{C}^{d}$ of $\Sigma(A, B, \Lambda)$ is given for $t \in[0, T]$ by

$$
x(t)=\sum_{\substack{(\mathbf{n}, j) \in \mathbb{N}^{N} \times \llbracket 1, N \rrbracket \\-\Lambda j \leq t-\Lambda \cdot \mathbf{n}<0}} \Xi_{\mathbf{n}-e_{j}} A_{j} x_{0}(t-\Lambda \cdot \mathbf{n})+\sum_{\substack{\mathbf{n} \in \mathbb{N}^{N} \\ \Lambda \cdot \mathbf{n} \leq t}} \Xi_{\mathbf{n}} B u(t-\Lambda \cdot \mathbf{n}) .
$$

Proposition 2.7 can be proved by verifying that the function $x:\left[-\Lambda_{\max }, T\right] \rightarrow \mathbb{C}^{d}$ defined in (2.3) satisfies indeed (1.1) for every $t \in[0, T]$ and is equal to the initial condition for negative time, which can be done by straightforward computations similar to the ones in [4, Lemma 3.13]. 
The controllability results we establish in Section 3 are based on the explicit representation for the solutions from Proposition 2.7. Notice that the control $u$ only affects the second term of (2.3). Since, in this term, $u$ is evaluated only at times $t-\Lambda \cdot \mathbf{n}$, one should pack together coefficients $\Xi_{\mathbf{n}}$ corresponding to different $\mathbf{n}, \mathbf{n}^{\prime} \in \mathbb{N}$ for which $\Lambda \cdot \mathbf{n}=\Lambda \cdot \mathbf{n}^{\prime}$, in the same manner as in [4, Definition 3.10].

Definition 2.8. Let $\Lambda=\left(\Lambda_{1}, \ldots, \Lambda_{N}\right) \in(0,+\infty)^{N}$. We partition $\mathbb{N}^{N}$ according to the equivalence relation $\sim$ defined by writing $\mathbf{n} \sim \mathbf{n}^{\prime}$ if $\Lambda \cdot \mathbf{n}=\Lambda \cdot \mathbf{n}^{\prime}$. We use $[\cdot]_{\Lambda}$ to denote the equivalence classes of $\sim$ and we set $\mathcal{N}_{\Lambda}=\mathbb{N}^{N} / \sim$. The index $\Lambda$ is omitted from the notation of $[\cdot]_{\Lambda}$ when the delay vector $\Lambda$ is clear from the context. We define

$$
\widehat{\Xi}_{[\mathbf{n}]}^{\Lambda}=\sum_{\mathbf{n}^{\prime} \in[\mathbf{n}]} \Xi_{\mathbf{n}^{\prime}}
$$

Thanks to Definition 2.8, the representation formula (2.3) for the solutions of $\Sigma(A, B, \Lambda)$ can be written as

$$
x(t)=\sum_{\substack{(\mathbf{n}, j) \in \mathbb{N}^{N} \times \llbracket 1, N \rrbracket \\-\Lambda_{j} \leq t-\Lambda \cdot \mathbf{n}<0}} \Xi_{\mathbf{n}-e_{j}} A_{j} x_{0}(t-\Lambda \cdot \mathbf{n})+\sum_{\substack{[\mathbf{n}] \in \mathcal{N}_{\Lambda} \\ \Lambda \cdot \mathbf{n} \leq t}} \widehat{\Xi}_{[\mathbf{n}]}^{\Lambda} B u(t-\Lambda \cdot \mathbf{n}) .
$$

\section{Relative controllability criteria}

This section presents the main relative controllability criteria from the paper, Theorems 3.1 and 3.2 below. Theorem 3.1 provides a criterion for relative controllability in the set of all functions and in the $L^{p}$ spaces, whereas the criterion in Theorem 3.2 characterizes relative controllability in the $\mathrm{e}^{k}$ spaces. Both algebraic criteria we obtain are expressed in terms of the coefficients $\widehat{\Xi}_{[\mathbf{n}]}^{\Lambda}$ and the matrix $B$ and are generalizations of the usual Kalman condition for the controllability of a discrete-time system. Their proofs are based on the explicit representation for solutions (2.5).

Theorem 3.1. Let $A=\left(A_{1}, \ldots, A_{N}\right) \in \mathcal{M}_{d}(\mathbb{C})^{N}, B \in \mathcal{M}_{d, m}(\mathbb{C}), \Lambda=\left(\Lambda_{1}, \ldots, \Lambda_{N}\right) \in(0,+\infty)^{N}, T>0$, and $p \in[1,+\infty]$. Define $\widehat{\mathbb{\Xi}}_{[\mathbf{n}]}^{\Lambda}$ as in (2.4). Then the following assertions are equivalent.

(a) One has

$$
\operatorname{Span}\left\{\widehat{\Xi}_{[\mathbf{n}]}^{\Lambda} B w \mid[\mathbf{n}] \in \mathcal{N}_{\Lambda}, \Lambda \cdot \mathbf{n} \leq T, w \in \mathbb{C}^{m}\right\}=\mathbb{C}^{d}
$$

(b) For every $x_{0}:\left[-\Lambda_{\max }, 0\right) \rightarrow \mathbb{C}^{d}$ and $x_{1} \in \mathbb{C}^{d}$, there exists $u:[0, T] \rightarrow \mathbb{C}^{m}$ such that the solution $x$ of $\Sigma(A, B, \Lambda)$ with initial condition $x_{0}$ and control u satisfies $x(T)=x_{1}$.

(c) There exists $\varepsilon_{0}>0$ such that, for every $\varepsilon \in\left(0, \varepsilon_{0}\right), x_{0}:\left[-\Lambda_{\max }, 0\right) \rightarrow \mathbb{C}^{d}$, and $x_{1}:[0, \varepsilon] \rightarrow \mathbb{C}^{d}$, there exists $u:[0, T+\varepsilon] \rightarrow \mathbb{C}^{m}$ such that the solution $x$ of $\Sigma(A, B, \Lambda)$ with initial condition $x_{0}$ and control $u$ satisfies $\left.x(T+\cdot)\right|_{[0, \varepsilon]}=x_{1}$.

(d) There exists $\varepsilon_{0}>0$ such that, for every $\varepsilon \in\left(0, \varepsilon_{0}\right), x_{0} \in L^{p}\left(\left(-\Lambda_{\max }, 0\right), \mathbb{C}^{d}\right)$, and $x_{1} \in L^{p}((0, \varepsilon)$, $\left.\mathbb{C}^{d}\right)$, there exists $u \in L^{p}\left((0, T+\varepsilon), \mathbb{C}^{m}\right)$ such that the solution $x$ of $\Sigma(A, B, \Lambda)$ with initial condition $x_{0}$ and control $u$ satisfies $x \in L^{p}\left(\left(-\Lambda_{\max }, T+\varepsilon\right), \mathbb{C}^{d}\right)$ and $\left.x(T+\cdot)\right|_{[0, \varepsilon]}=x_{1}$.

Proof. For $T>0$, let $\mathcal{N}^{T}=\left\{[\mathbf{n}] \in \mathcal{N}_{\Lambda} \mid \Lambda \cdot \mathbf{n} \leq T\right\}$ and $n_{T}=\# \mathcal{N}^{T}$. The proof is carried out as follows. Clearly, (c) $\Longrightarrow$ (b). We will show the equivalences by proving that (b) $\Longrightarrow$ (a), (a) $\Longrightarrow$ (c) and (d), and (d) $\Longrightarrow$ (a).

Assume that (b) is satisfied, which shows, using (2.5) and considering a zero initial condition, that, for every $x_{1} \in \mathbb{C}^{d}$, there exists $u:[0, T] \rightarrow \mathbb{C}^{m}$ such that

$$
\left(\widehat{\Xi}_{[\mathbf{n}]}^{\Lambda} B\right)_{[\mathbf{n}] \in \mathcal{N}^{T}}(u(T-\Lambda \cdot \mathbf{n}))_{[\mathbf{n}] \in \mathcal{N}^{T}}=\sum_{[\mathbf{n}] \in \mathcal{N}^{T}} \widehat{\Xi}_{[\mathbf{n}]}^{\Lambda} B u(T-\Lambda \cdot \mathbf{n})=x_{1},
$$


where $\left(\widehat{\Xi}_{[\mathbf{n}]}^{\Lambda} B\right)_{[\mathbf{n}] \in \mathcal{N} T}$ denotes the $d \times m n_{T}$ matrix composed of the $n_{T}$ blocks $\widehat{\Xi}_{[\mathbf{n}]}^{\Lambda} B$ of size $d \times m$ and $(u(T-\Lambda \cdot \mathbf{n}))_{[\mathbf{n}] \in \mathcal{N}^{T}}$ denotes the $m n_{T} \times 1$ matrix composed of the $n_{T}$ blocks $u(T-\Lambda \cdot \mathbf{n})$ of size $m \times 1$. This means that the map $\mathbb{C}^{m n_{T}} \ni U \mapsto\left(\widehat{\Xi}_{[\mathbf{n}]}^{\Lambda} B\right)_{[\mathbf{n}] \in \mathcal{N}^{T}} U \in \mathbb{C}^{d}$ is surjective, and thus (a) is satisfied.

Assume now that (a) is satisfied and let

$$
\varepsilon_{0}=\min \left\{\min _{\substack{\left[\mathbf{n}^{\prime}\right],[\mathbf{n}] \in \mathcal{N}^{T} \\\left[\mathbf{n}^{\prime}\right] \neq[\mathbf{n}]}}\left|\Lambda \cdot \mathbf{n}-\Lambda \cdot \mathbf{n}^{\prime}\right|, \min _{\substack{\mathbf{n} \in \mathbb{N}^{N} \\ \Lambda \cdot \mathbf{n}>T}}(\Lambda \cdot \mathbf{n}-T)\right\}>0 .
$$

Let $\varepsilon \in\left(0, \varepsilon_{0}\right), x_{0}:\left[-\Lambda_{\max }, 0\right) \rightarrow \mathbb{C}^{d}$, and $x_{1}:[0, \varepsilon] \rightarrow \mathbb{C}^{d}$. Thanks to (a), the map $\mathbb{C}^{m n_{T}} \ni U \mapsto$ $\left(\widehat{\Xi}_{[\mathbf{n}]^{\Lambda}}^{\Lambda} B\right)_{[\mathbf{n}] \in \mathcal{N}^{T}} U \in \mathbb{C}^{d}$ is surjective, and hence the $d \times m n_{T}$ matrix $\left(\widehat{\Xi}_{[\mathbf{n}]}^{\Lambda} B\right)_{[\mathbf{n}] \in \mathcal{N}^{T}}$ admits a right inverse $M \in \mathcal{M}_{m n_{T}, d}(\mathbb{C})$. Let $U=\left(U_{[\mathbf{n}]}\right)_{[\mathbf{n}] \in \mathcal{N}^{T}}:[0, \varepsilon] \rightarrow \mathbb{C}^{m n_{T}}=\left(\mathbb{C}^{m}\right)^{\mathcal{N}^{T}}$ be given by

$$
U(t)=M\left(x_{1}(t)-\sum_{\substack{(\mathbf{n}, j) \in \mathbb{N}^{N} \times \llbracket 1, N \rrbracket \\-\Lambda_{j} \leq T+t-\Lambda \cdot \mathbf{n}<0}} \Xi_{\mathbf{n}-e_{j}} A_{j} x_{0}(T+t-\Lambda \cdot \mathbf{n})\right) .
$$

Define $u:[0, T+\varepsilon] \rightarrow \mathbb{C}^{m}$ by

$$
u(t)= \begin{cases}U_{[\mathbf{n}]}(\Lambda \cdot \mathbf{n}+t-T), & \text { if } t \in[T-\Lambda \cdot \mathbf{n}, T-\Lambda \cdot \mathbf{n}+\varepsilon] \text { for some }[\mathbf{n}] \in \mathcal{N}^{T}, \\ 0, & \text { otherwise. }\end{cases}
$$

Thanks to the definition of $\varepsilon_{0}, u$ is well-defined, and one has $u(T+t-\Lambda \cdot \mathbf{n})=U_{[\mathbf{n}]}(t)$ for every $[\mathbf{n}] \in \mathcal{N}^{T}$ and $t \in[0, \varepsilon]$. Hence, it follows from (3.3) that, for every $t \in[0, \varepsilon]$,

$$
\begin{array}{r}
x_{1}(t)-\sum_{\substack{(\mathbf{n}, j) \in \mathbb{N}^{N} \times \llbracket 1, N \rrbracket \\
-\Lambda j \leq T+t-\Lambda \cdot \mathbf{n}<0}} \Xi_{\mathbf{n}-e_{j}} A_{j} x_{0}(T+t-\Lambda \cdot \mathbf{n})=\left(\widehat{\Xi}_{[\mathbf{n}]}^{\Lambda} B\right)_{[\mathbf{n}] \in \mathcal{N}^{T}}(u(T+t-\Lambda \cdot \mathbf{n}))_{[\mathbf{n}] \in \mathcal{N} T} \\
=\sum_{[\mathbf{n}] \in \mathcal{N} T} \widehat{\Xi}_{[\mathbf{n}]}^{\Lambda} B u(T+t-\Lambda \cdot \mathbf{n})=\sum_{\substack{[\mathbf{n}] \in \mathcal{N}_{\Lambda} \\
\Lambda \cdot \mathbf{n} \leq T+t}} \widehat{\Xi}_{[\mathbf{n}]}^{\Lambda} B u(T+t-\Lambda \cdot \mathbf{n}),
\end{array}
$$

where we use that, thanks to the definition of $\varepsilon_{0}$, one has

$$
\mathcal{N}^{T}=\left\{[\mathbf{n}] \in \mathcal{N}_{\Lambda} \mid \Lambda \cdot \mathbf{n} \leq T+t\right\}, \quad \forall t \in[0, \varepsilon] .
$$

It now follows from (2.5) and (3.5) that the solution $x$ of $\Sigma(A, B, \Lambda)$ with initial condition $x_{0}$ and control $u$ satisfies $\left.x(T+\cdot)\right|_{[0, \varepsilon]}=x_{1}$, and hence (c) holds. Notice moreover that, if we assume $x_{0} \in$ $L^{p}\left(\left(-\Lambda_{\max }, 0\right), \mathbb{C}^{d}\right)$ and $x_{1} \in L^{p}\left((0, \varepsilon), \mathbb{C}^{d}\right)$, it follows from (3.3) that $U \in L^{p}\left((0, \varepsilon), \mathbb{C}^{m n_{T}}\right)$, and thus, by (3.4), $u \in L^{p}\left((0, T+\varepsilon), \mathbb{C}^{m}\right)$. Hence, the solution $x$ of $\Sigma(A, B, \Lambda)$ with initial condition $x_{0}$ and control $u$ satisfies $x \in L^{p}\left(\left(-\Lambda_{\max }, T+\varepsilon\right), \mathbb{C}^{d}\right)$, thanks to Remark 2.3, and $\left.x(T+\cdot)\right|_{[0, \varepsilon]}=x_{1}$, which shows that (d) also holds.

Finally, assume that (d) holds, take $\varepsilon_{0}>0$ as in (d) and fix $\varepsilon \in\left(0, \varepsilon_{0}\right)$. Then, considering a zero initial condition, for every constant final state $x_{1} \in \mathbb{C}^{d}$, there exists $u \in L^{p}\left((0, T+\varepsilon), \mathbb{C}^{m}\right)$ such that, for almost every $t \in(0, \varepsilon)$, one has, as in (3.2),

$$
\left(\widehat{\Xi}_{[\mathbf{n}]}^{\Lambda} B\right)_{[\mathbf{n}] \in \mathcal{N}^{T}}(u(T+t-\Lambda \cdot \mathbf{n}))_{[\mathbf{n}] \in \mathcal{N}^{T}}=x_{1},
$$

where we use that (3.6) holds, up to choosing a smaller $\varepsilon \in\left(0, \varepsilon_{0}\right)$. Hence, as in (3.2), one also obtains that the map $\mathbb{C}^{m n_{T}} \ni U \mapsto\left(\widehat{\Xi}_{[\mathbf{n}]}^{\Lambda} B\right)_{[\mathbf{n}] \in \mathcal{N} T} U \in \mathbb{C}^{d}$ is surjective, and thus (a) is satisfied. 
The next result presents a relative controllability criterion for $\mathrm{C}^{k}$ solutions of $\Sigma(A, B, \Lambda)$, which is slightly different from (a) in Theorem 3.1 due to the compatibility condition (2.1) required for the existence of $\mathrm{e}^{k}$ solutions.

Theorem 3.2. Let $A=\left(A_{1}, \ldots, A_{N}\right) \in \mathcal{M}_{d}(\mathbb{C})^{N}, B \in \mathcal{M}_{d, m}(\mathbb{C}), \Lambda=\left(\Lambda_{1}, \ldots, \Lambda_{N}\right) \in(0,+\infty)^{N}, T>0$, and $k \in \mathbb{N}$. Define $\widehat{\mathbb{\Xi}}_{[\mathbf{n}]}^{\Lambda}$ as in (2.4). Then the following assertions are equivalent.

(a) One has

$$
\operatorname{Span}\left\{\widehat{\Xi}_{[\mathbf{n}]}^{\Lambda} B w \mid[\mathbf{n}] \in \mathcal{N}_{\Lambda}, \Lambda \cdot \mathbf{n}<T, w \in \mathbb{C}^{m}\right\}=\mathbb{C}^{d}
$$

(b) For every $x_{0} \mathrm{e}^{k}$-admissible for $\Sigma(A, B, \Lambda)$ and $x_{1} \in \mathbb{C}^{d}$, there exists $u \in \mathrm{e}^{k}\left([0, T], \mathbb{C}^{m}\right)$ such that the solution $x$ of $\Sigma(A, B, \Lambda)$ with initial condition $x_{0}$ and control $u$ satisfies $x \in \mathcal{C}^{k}\left(\left[-\Lambda_{\max }, T\right]\right.$, $\left.\mathbb{C}^{d}\right)$ and $x(T)=x_{1}$.

(c) There exists $\varepsilon_{0}>0$ such that, for every $\varepsilon \in\left(0, \varepsilon_{0}\right), x_{0} \mathrm{e}^{k}$-admissible for $\Sigma(A, B, \Lambda)$, and $x_{1} \in$ $\mathrm{C}^{k}\left([0, \varepsilon], \mathbb{C}^{d}\right)$, there exists $u \in \mathcal{C}^{k}\left([0, T+\varepsilon], \mathbb{C}^{m}\right)$ such that the solution $x$ of $\Sigma(A, B, \Lambda)$ with initial condition $x_{0}$ and control $u$ satisfies $x \in \mathrm{C}^{k}\left(\left[-\Lambda_{\max }, T+\varepsilon\right], \mathbb{C}^{d}\right)$ and $\left.x(T+\cdot)\right|_{[0, \varepsilon]}=x_{1}$.

Proof. Let $\mathcal{N}_{*}^{T}=\left\{[\mathbf{n}]_{\Lambda} \in \mathcal{N}_{\Lambda} \mid \Lambda \cdot \mathbf{n}<T\right\}$ and $n_{T}^{*}=\# \mathcal{N}_{*}^{T}$. We begin the proof by noticing that (c) implies (b). Assume now that (b) holds and let us show that (a) is satisfied. For every $x_{1} \in \mathbb{C}^{d}$, there exists $u \in \mathcal{C}^{k}\left([0, T], \mathbb{C}^{m}\right)$ such that the solution $x$ of $\Sigma(A, B, \Lambda)$ with zero initial condition and control $u$ satisfies $x \in \mathrm{C}^{k}\left(\left[-\Lambda_{\max }, T\right], \mathbb{C}^{d}\right)$ and, from (2.5),

$$
\sum_{\substack{[\mathbf{n}] \in \mathcal{N}_{\Lambda} \\ \Lambda \cdot \mathbf{n} \leq T}} \widehat{\Xi}_{[\mathbf{n}]}^{\Lambda} B u(T-\Lambda \cdot \mathbf{n})=x_{1} .
$$

Moreover, since $x \in \mathfrak{C}^{k}\left(\left[-\Lambda_{\max }, T\right], \mathbb{C}^{d}\right)$, it follows from Remark 2.4 that (2.1) is satisfied, and thus, for every $r \in \llbracket 0, k \rrbracket, B u^{(r)}(0)=0$. Thus (3.8) becomes

$$
\sum_{\substack{[\mathbf{n}] \in \mathcal{N}_{\Lambda} \\ \Lambda \cdot \mathbf{n}<T}} \widehat{\Xi}_{[\mathbf{n}]}^{\Lambda} B u(T-\Lambda \cdot \mathbf{n})=x_{1},
$$

and we conclude, as in the proof of Theorem 3.1, that $\mathbb{C}^{m n_{T}^{*}} \ni U \mapsto\left(\widehat{\Xi}_{[\mathbf{n}]}^{\Lambda} B\right)_{[\mathbf{n}] \in \mathcal{N}_{*}^{T}} U \in \mathbb{C}^{d}$ is surjective, and thus (a) is satisfied.

Finally, assume that (a) is satisfied and let

$$
\varepsilon_{0}=\frac{1}{2} \min \left\{\min _{\substack{\left[\mathbf{n}^{\prime}\right],[\mathbf{n}] \in \mathcal{N}_{*}^{T} \\\left[\mathbf{n}^{\prime}\right] \neq[\mathbf{n}]}}\left|\Lambda \cdot \mathbf{n}-\Lambda \cdot \mathbf{n}^{\prime}\right|, \min _{\substack{\mathbf{n} \in \mathbb{N}^{N} \\ \Lambda \cdot \mathbf{n} \neq T}}|\Lambda \cdot \mathbf{n}-T|\right\}>0
$$

Let $\varepsilon \in\left(0, \varepsilon_{0}\right), x_{0} \mathcal{C}^{k}$-admissible for $\Sigma(A, B, \Lambda)$, and $x_{1} \in \mathcal{C}^{k}\left([0, \varepsilon], \mathbb{C}^{d}\right)$. Since $x_{0}$ is $\mathrm{C}^{k}$-admissible, there exists $\mu \in \mathrm{C}^{k}\left([0, \varepsilon], \mathbb{C}^{m}\right)$, with a compact support inside $[0, \varepsilon)$, such that, for every $r \in \llbracket 0, k \rrbracket$,

$$
\lim _{t \rightarrow 0} x_{0}^{(r)}(t)=\sum_{j=1}^{N} A_{j} x_{0}^{(r)}\left(-\Lambda_{j}\right)+B \mu^{(r)}(0) .
$$

If $T=\Lambda \cdot \mathbf{n}$ for some $\mathbf{n} \in \mathbb{N}^{N}$, we set $\delta_{T}=1$ and $\tau=[\mathbf{n}]$; otherwise, we set $\delta_{T}=0$ and $\tau=[0]$. As in the proof of Theorem 3.1, it follows from (a) that the $d \times m n_{T}^{*}$ matrix $\left(\widehat{\Xi}_{[\mathbf{n}]}^{\Lambda} B\right)_{[\mathbf{n}] \in \mathcal{N}_{*}^{T}}$ admits a right 
inverse $M \in \mathcal{M}_{m n_{T}^{*}, d}(\mathbb{C})$. Let $U=\left(U_{[\mathbf{n}]}\right)_{[\mathbf{n}] \in \mathcal{N}_{*}^{T}}:[0, \varepsilon] \rightarrow \mathbb{C}^{m n_{T}^{*}}=\left(\mathbb{C}^{m}\right)^{\mathcal{N}_{*}^{T}}$ be given by

$$
U(t)=M\left(x_{1}(t)-\sum_{\substack{(\mathbf{n}, j) \in \mathbb{N}^{N} \times \llbracket 1, N \rrbracket \\-\Lambda, j \leq T+t-\Lambda \cdot \mathbf{n}<0}} \Xi_{\mathbf{n}-e_{j}} A_{j} x_{0}(T+t-\Lambda \cdot \mathbf{n})-\delta_{T} \widehat{\Xi}_{\tau}^{\Lambda} B \mu(t)\right) .
$$

Notice that the sum in (3.10) can be taken over the set

$$
G_{1}(t)=\left\{\left(\mathbf{n}=\left(n_{1}, \ldots, n_{N}\right), j\right) \in \mathbb{N}^{N} \times \llbracket 1, N \rrbracket \mid-\Lambda_{j} \leq T+t-\Lambda \cdot \mathbf{n}<0, n_{j} \geq 1\right\},
$$

since $\Xi_{\mathbf{n}}=0$ if $\mathbf{n} \in \mathbb{Z}^{N} \backslash \mathbb{N}^{N}$. Moreover, thanks to the definition of $\varepsilon_{0}$, one has $G_{1}(t)=G_{1}(0)$ for every $t \in[0, \varepsilon]$, and thus $U$ can be written for $t \in[0, \varepsilon]$ as

$$
U(t)=M\left(x_{1}(t)-\sum_{\substack{(\mathbf{n}, j) \in \mathbb{N}^{N} \times \llbracket 1, N \rrbracket \\-\Lambda, j \leq T-\Lambda \cdot \mathbf{n}<0}} \Xi_{\mathbf{n}-e_{j}} A_{j} x_{0}(T+t-\Lambda \cdot \mathbf{n})-\delta_{T} \widehat{\Xi}_{\tau}^{\Lambda} B \mu(t)\right) .
$$

In particular, one obtains that $U \in \mathcal{C}^{k}\left([0, \varepsilon], \mathbb{C}^{m n_{T}^{*}}\right)$. We extend $U$ into a $\mathrm{C}^{k}$ function on the interval $\left[-\frac{\varepsilon}{2}, \frac{3 \varepsilon}{2}\right]$ with a compact support in $\left(-\frac{\varepsilon}{2}, \frac{3 \varepsilon}{2}\right)$. Define $u:[0, T+\varepsilon] \rightarrow \mathbb{C}^{m}$ by

$$
u(t)= \begin{cases}U_{[\mathbf{n}]}(\Lambda \cdot \mathbf{n}+t-T), & \text { if } t \in\left[T-\Lambda \cdot \mathbf{n}-\frac{\varepsilon}{2}, T-\Lambda \cdot \mathbf{n}+\frac{3 \varepsilon}{2}\right] \text { for some }[\mathbf{n}] \in \mathcal{N}_{*}^{T}, \\ \mu(t), & \text { if } t \in[0, \varepsilon], \\ 0, & \text { otherwise, }\end{cases}
$$

which is well-defined thanks to the choice of $\varepsilon_{0}$, and satisfies $u \in \mathfrak{C}^{k}\left([0, T+\varepsilon], \mathbb{C}^{m}\right)$ thanks to the construction of $U$ and $\mu$. Moreover, one has $u(T+t-\Lambda \cdot \mathbf{n})=U_{[\mathbf{n}]}(t)$ for every $[\mathbf{n}] \in \mathcal{N}_{*}^{T}$ and, thanks to (3.9), it follows from Remark 2.4 that the unique solution $x$ of $\Sigma(A, B, \Lambda)$ with initial condition $x_{0}$ and control $u$ satisfies $x \in \mathcal{C}^{k}\left(\left[-\Lambda_{\max }, T+\varepsilon\right], \mathbb{C}^{d}\right)$. It follows from (3.10) that, for every $t \in[0, \varepsilon]$,

$$
\begin{aligned}
& x_{1}(t)-\sum_{\substack{(\mathbf{n}, j) \in \mathbb{N}^{N} \times \llbracket 1, N \rrbracket \\
-\Lambda, \leq T+t-\Lambda \cdot \mathbf{n}<0}} \Xi_{\mathbf{n}-e_{j}} A_{j} x_{0}(T+t-\Lambda \cdot \mathbf{n}) \\
= & \delta_{T} \widehat{\Xi}_{\tau}^{\Lambda} B \mu(t)+\left(\widehat{\Xi}_{[\mathbf{n}]}^{\Lambda} B\right)_{[\mathbf{n}] \in \mathcal{N}_{*}^{T}}(u(T+t-\Lambda \cdot \mathbf{n}))_{[\mathbf{n}] \in \mathcal{N}_{*}^{T}} \\
= & \sum_{\substack{[\mathbf{n}] \in \mathcal{N}_{\Lambda} \\
\Lambda \cdot \mathbf{n} \leq T}} \widehat{\Xi}_{[\mathbf{n}]}^{\Lambda} B u(T+t-\Lambda \cdot \mathbf{n})=\sum_{\substack{[\mathbf{n}] \in \mathcal{N}_{\Lambda} \\
\Lambda \cdot \mathbf{n} \leq T+t}} \widehat{\Xi}_{[\mathbf{n}]}^{\Lambda} B u(T+t-\Lambda \cdot \mathbf{n}),
\end{aligned}
$$

and thus one obtains that the solution $x$ of $\Sigma(A, B, \Lambda)$ with initial condition $x_{0}$ and control $u$ satisfies $\left.x(T+\cdot)\right|_{[0, \varepsilon]}=x_{1}$, which shows that (c) holds.

Remark 3.3. When $N=1$, the controlled difference equation (1.1) becomes $x(t)=A x(t-\Lambda)+B u(t)$, with $A=A_{1}$ and $\Lambda=\Lambda_{1}$. It follows from Definitions 2.6 and 2.8 that, for $\mathbf{n}=n \in \mathbb{N}$, one has $\widehat{\Xi_{[\mathbf{n}]}}=A^{n}$, and thus condition (a) from Theorem 3.1 reduces to $\operatorname{rk}\left(\begin{array}{lllll}B & A B & A^{2} B & \cdots & A^{\lfloor T / \Lambda\rfloor} B\end{array}\right)=d$, which is the usual Kalman condition for controllability of discrete-time linear systems (see, e.g., [33, Theorem

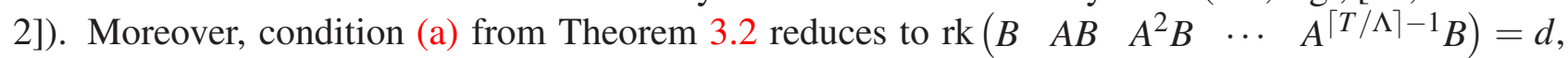
which is the same as the previous one when $T / \Lambda \notin \mathbb{N}^{*}$.

Notice that (b), (c), and (d) from Theorem 3.1 and (b) and (c) from Theorem 3.2 could all be used to define relative controllability in different function spaces. Motivated by the equivalences established in Theorems 3.1 and 3.2, we provide the following definition. 
Definition 3.4. Let $A=\left(A_{1}, \ldots, A_{N}\right) \in \mathcal{M}_{d}(\mathbb{C})^{N}, B \in \mathcal{M}_{d, m}(\mathbb{C}), \Lambda \in(0,+\infty)^{N}$, and $T>0$.

(a) We say that $\Sigma(A, B, \Lambda)$ is relatively controllable in time $T$ if

$$
\operatorname{Span}\left\{\widehat{\Xi}_{[\mathbf{n}]}^{\Lambda} B w \mid[\mathbf{n}] \in \mathcal{N}_{\Lambda}, \Lambda \cdot \mathbf{n} \leq T, w \in \mathbb{C}^{m}\right\}=\mathbb{C}^{d} .
$$

(b) If $\Sigma(A, B, \Lambda)$ is relatively controllable in some time $T>0$, we define the minimal controllability time $T_{\min }$ for $\Sigma(A, B, \Lambda)$ by $T_{\min }=\inf \{T>0 \mid \Sigma(A, B, \Lambda)$ is relativelycontrollable in time $T\}$.

Remark 3.5. Contrarily to the situation for linear control systems of the form $\dot{x}(t)=A x(t)+B u(t)$ or $x(t)=A x(t-1)+B u(t)$, relative controllability for some time $T>0$ does not imply stabilizability by a linear feedback law. Indeed [17, Theorem 3.1] proves that $\Sigma(A, B, \Lambda)$ can be strongly stabilized by a linear feedback law $u(t)=\sum_{j=1}^{N} K_{j} x\left(t-\Lambda_{j}\right)$ if and only if there exists $\varepsilon>0$ such that, for every $\lambda \in \mathbb{C}$ with $\operatorname{Re} \lambda \geq-\varepsilon$, one has

$$
\operatorname{rk}\left(B \quad \operatorname{Id}_{d}-\sum_{j=1}^{N} A_{j} e^{-\lambda \Lambda_{j}}\right)=d .
$$

For $N=d=2$ and $m=1$, consider the system $\Sigma(A, B, \Lambda)$ with $A=\left(A_{1}, A_{2}\right), B$, and $\Lambda=\left(\Lambda_{1}, \Lambda_{2}\right)$ given by

$$
\begin{array}{ll}
A_{1}=\left(\begin{array}{cc}
\alpha & -\alpha^{1-\ell} \\
0 & 0
\end{array}\right), & A_{2}=\left(\begin{array}{ll}
0 & 1 \\
0 & 0
\end{array}\right), \\
\Lambda_{1}=1, & \Lambda_{2}=\ell,
\end{array}
$$

with $\ell \in(0,1)$ and $\alpha>1$. Clearly, $\Sigma(A, B, \Lambda)$ is relatively controllable in time $T \geq \ell$ since $\operatorname{Span}\{B$, $\left.A_{2} B\right\}=\mathbb{C}^{2}$. However, for $\lambda \in \mathbb{C}$, one has

$$
\mathrm{Id}_{2}-A_{1} e^{-\lambda}-A_{2} e^{-\lambda \ell}=\left(\begin{array}{cc}
1-\alpha e^{-\lambda} & \alpha^{1-\ell} e^{-\lambda}-e^{-\lambda \ell} \\
0 & 1
\end{array}\right),
$$

and the first row of this matrix is zero for $\lambda=\ln \alpha$. Hence (3.11) does not hold for $\lambda=\ln \alpha>0$, which shows in particular that $\Sigma(A, B, \Lambda)$ cannot be strongly stabilized by a linear feedback law.

\section{Rational dependence of the delays}

This section compares relative controllability of $\Sigma(A, B, \Lambda)$ for different delay vectors $\Lambda$ in terms of their rational dependence structure. We start by recalling the definition of rational dependence and commensurability.

Definition 4.1. Let $\Lambda=\left(\Lambda_{1}, \ldots, \Lambda_{N}\right) \in \mathbb{R}^{N}$.

(a) We say that the components of $\Lambda$ are rationally dependent if there exists $\mathbf{n} \in \mathbb{Z}^{N} \backslash\{0\}$ such that $\Lambda \cdot \mathbf{n}=0$. Otherwise, the components of $\Lambda$ are said to be rationally independent .

(b) We say that the components of $\Lambda$ are commensurable if there exist $\lambda \in \mathbb{R}$ and $k \in \mathbb{Z}^{N}$ such that $\Lambda=\lambda k$.

Notice that the set $\mathbb{Z}^{N}$ can be replaced by $\mathbb{Q}^{N}$ in Definition 4.1 without changing the definitions of rational dependence and commensurability. We next introduce a preorder in the set of all possible delay vectors $(0,+\infty)^{N}$, which describes when one delay vector is "less rationally dependent" than another. 
Definition 4.2. For $\Lambda \in(0,+\infty)^{N}$, we define $Z(\Lambda)=\left\{\mathbf{n} \in \mathbb{Z}^{N} \mid \Lambda \cdot \mathbf{n}=0\right\}$. For $\Lambda, L \in(0,+\infty)^{N}$, we write $\Lambda \preccurlyeq L$ or, equivalently, $L \succcurlyeq \Lambda$, if $Z(\Lambda) \subset Z(L)$. We write $\Lambda \approx L$ if $\Lambda \preccurlyeq L$ and $L \preccurlyeq \Lambda$.

If $\Lambda \in(0,+\infty)^{N}$ has rationally independent components, then one immediately computes $Z(\Lambda)=$ $\{0\}$, and hence $\Lambda \preccurlyeq L$ for every $L \in(0,+\infty)^{N}$, that is, delay vectors with rationally independent components are minimal for the preorder $\preccurlyeq$. Notice also that, for $\Lambda \in(0,+\infty)^{N}$, the set $Z(\Lambda)$ encodes the structure of the equivalence classes $[\mathbf{n}]_{\Lambda}$ for $\mathbf{n} \in \mathbb{N}^{N}$, in the sense that, for $\mathbf{n}^{\prime} \in \mathbb{N}^{N}$, one has $\mathbf{n}^{\prime} \in[\mathbf{n}]_{\Lambda}$ if and only if $\mathbf{n}^{\prime}-\mathbf{n} \in Z(\Lambda)$, which shows that $[\mathbf{n}]_{\Lambda}=(\mathbf{n}+Z(\Lambda)) \cap \mathbb{N}^{N}$. We recall the following result from [4].

Proposition 4.3 ([4], Proposition 3.9). Let $\Lambda=\left(\Lambda_{1}, \ldots, \Lambda_{N}\right) \in(0,+\infty)^{N}$. There exist $h \in \llbracket 1, N \rrbracket$, $\ell=\left(\ell_{1}, \ldots, \ell_{h}\right) \in(0,+\infty)^{h}$ with rationally independent components, and $M \in \mathcal{M}_{N, h}(\mathbb{N})$ with $\mathrm{rk} M=h$ such that $\Lambda=M \ell$. Moreover, for every $M$ as before, one has

$$
\operatorname{Ran} M=\left\{L \in \mathbb{R}^{N} \mid \text { for every } \mathbf{n} \in Z(\Lambda) \text {, one has } L \cdot \mathbf{n}=0\right\} .
$$

In particular, it follows from Proposition 4.3 that the set of all $L \in(0,+\infty)^{N}$ such that $L \succcurlyeq \Lambda$ is $\operatorname{Ran} M \cap(0,+\infty)^{N}$. The next proposition gathers some immediate properties that follow from Definition 4.2 .

Proposition 4.4. Let $\Lambda, L \in(0,+\infty)^{N}$. If $\Lambda \preccurlyeq L$, then, for every $\mathbf{n} \in \mathbb{N}^{N}$, one has $[\mathbf{n}]_{\Lambda} \subset[\mathbf{n}]_{L}$ and

$$
\widehat{\boldsymbol{\Xi}}_{[\mathbf{n}]}^{L}=\sum_{\substack{\tau \in \mathcal{N}_{\Lambda} \\ \tau \subset[\mathbf{n}]_{L}}} \widehat{\boldsymbol{\Xi}}_{\tau}^{\Lambda} .
$$

In particular, if $\Lambda \approx L$, then, for every $\mathbf{n} \in \mathbb{N}^{N}$, one has $[\mathbf{n}]_{\Lambda}=[\mathbf{n}]_{L}$ and $\widehat{\Xi}_{[\mathbf{n}]}^{\Lambda}=\widehat{\Xi}_{[\mathbf{n}]}^{L}$.

Proof. If $\Lambda \preccurlyeq L$ and $\mathbf{n} \in \mathbb{N}^{N}$, the inclusion $[\mathbf{n}]_{\Lambda} \subset[\mathbf{n}]_{L}$ follows immediately from the fact that $Z(\Lambda) \subset$ $Z(L)$ and that $[\mathbf{n}]_{\lambda}=(\mathbf{n}+Z(\lambda)) \cap \mathbb{N}^{N}$ for every $\mathbf{n} \in \mathbb{N}^{N}$ and $\lambda \in(0,+\infty)^{N}$. Moreover, the set $\{\tau \in$ $\left.\mathcal{N}_{\Lambda} \mid \tau \subset[\mathbf{n}]_{L}\right\}$ is a partition of $[\mathbf{n}]_{L}$, since, for every $\mathbf{n}^{\prime} \in[\mathbf{n}]_{L}$, one has $\left[\mathbf{n}^{\prime}\right]_{\Lambda} \subset\left[\mathbf{n}^{\prime}\right]_{L}=[\mathbf{n}]_{L}$ and all equivalence classes in $\mathcal{N}_{\Lambda}$ are disjoint. Hence

$$
\sum_{\substack{\tau \in \mathcal{N}_{\Lambda} \\ \tau \subset[\mathbf{n}]_{L}}} \widehat{\Xi}_{\tau}^{\Lambda}=\sum_{\substack{\tau \in \mathcal{N}_{\Lambda} \\ \tau \subset[\mathbf{n}]_{L}}} \sum_{\mathbf{n}^{\prime} \in \tau} \Xi_{\mathbf{n}^{\prime}}=\sum_{\mathbf{n}^{\prime} \in[\mathbf{n}]_{L}} \Xi_{\mathbf{n}^{\prime}}=\widehat{\Xi}_{[\mathbf{n}]}^{L} .
$$

The statements in the case $\Lambda \approx L$ follow immediately.

The first main result of this section is the following theorem.

Theorem 4.5. Let $A=\left(A_{1}, \ldots, A_{N}\right) \in \mathcal{M}_{d}(\mathbb{C})^{N}, B \in \mathcal{M}_{d, m}(\mathbb{C}), \Lambda, L \in(0,+\infty)^{N}$, and $T>0$ be such

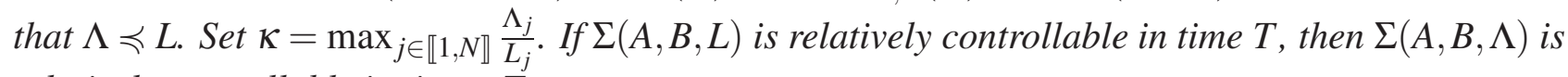
relatively controllable in time $\kappa T$.

Proof. Notice that, for every $\mathbf{n}=\left(n_{1}, \ldots, n_{N}\right) \in \mathbb{N}^{N} \backslash\{0\}$, one has $\frac{\Lambda \cdot \mathbf{n}}{L \cdot \mathbf{n}}=\sum_{j=1}^{N} \frac{\Lambda_{j}}{L_{j}} \frac{L_{j} n_{j}}{L \cdot \mathbf{n}} \leq \kappa$, and thus $\Lambda \cdot \mathbf{n} \leq \kappa L \cdot \mathbf{n}$ for every $\mathbf{n} \in \mathbb{N}^{N}$. Using Proposition 4 .4, one obtains that

$$
\begin{aligned}
& \operatorname{Span}\left\{\widehat{\Xi}_{[\mathbf{n}]}^{L} B w \mid[\mathbf{n}] \in \mathcal{N}_{L}, L \cdot \mathbf{n} \leq T, w \in \mathbb{C}^{m}\right\} \\
= & \operatorname{Span}\left\{\sum_{\substack{\tau \in \mathcal{N}_{\Lambda} \\
\tau \subset[\mathbf{n}]_{L}}} \widehat{\Xi}_{\tau}^{\Lambda} B w \mid[\mathbf{n}] \in \mathcal{N}_{L}, L \cdot \mathbf{n} \leq T, w \in \mathbb{C}^{m}\right\}
\end{aligned}
$$




$$
\begin{aligned}
& \subset \operatorname{Span}\left\{\widehat{\Xi}_{\tau}^{\Lambda} B w \mid \tau \in \mathcal{N}_{\Lambda}, \tau \subset[\mathbf{n}]_{L},[\mathbf{n}]_{L} \in \mathcal{N}_{L}, L \cdot \mathbf{n} \leq T, w \in \mathbb{C}^{m}\right\} \\
& =\operatorname{Span}\left\{\widehat{\mathbb{\Xi}}_{[\mathbf{n}]}^{\Lambda} B w \mid[\mathbf{n}] \in \mathcal{N}_{\Lambda}, L \cdot \mathbf{n} \leq T, w \in \mathbb{C}^{m}\right\} \\
& \subset \operatorname{Span}\left\{\widehat{\mathbb{\Xi}}_{[\mathbf{n}]}^{\Lambda} B w \mid[\mathbf{n}] \in \mathcal{N}_{\Lambda}, \Lambda \cdot \mathbf{n} \leq \kappa T, w \in \mathbb{C}^{m}\right\},
\end{aligned}
$$

which proves the statement.

Theorem 4.5 proves that relative controllability of $\Sigma(A, B, L)$ implies that of $\Sigma(A, B, \Lambda)$ for all delay vectors $\Lambda$ such that $\Lambda \preccurlyeq L$ (with different controllability times). The converse of this result does not hold, as illustrated in the following example.

Example 4.6. Consider the system $\Sigma(A, B, \Lambda)$ with $N=2, d=3, m=1, \Lambda=(1, \lambda)$ for some $\lambda \in$ $(0,1)$, and

$$
A_{1}=\left(\begin{array}{ccc}
0 & 0 & -1 \\
0 & 0 & 0 \\
0 & 0 & 0
\end{array}\right), \quad A_{2}=\left(\begin{array}{ccc}
0 & 1 & 0 \\
0 & 0 & 1 \\
0 & 0 & 0
\end{array}\right), \quad B=\left(\begin{array}{l}
0 \\
0 \\
1
\end{array}\right)
$$

One has $A_{1}=-A_{2}^{2}$ and hence one immediately computes

$$
\Xi_{\mathbf{n}}= \begin{cases}\mathrm{Id}_{3}, & \text { if } \mathbf{n}=(0,0), \\ A_{1}, & \text { if } \mathbf{n}=(1,0), \\ A_{2}, & \text { if } \mathbf{n}=(0,1), \\ A_{2}^{2}, & \text { if } \mathbf{n}=(0,2), \\ 0, & \text { otherwise }\end{cases}
$$

If $\lambda \notin \mathbb{Q}$, one has $\widehat{\Xi}_{[\mathbf{n}]}^{\Lambda}=\Xi_{\mathbf{n}}$ for every $\mathbf{n} \in \mathbb{N}^{2}$, and thus, for every $T \geq 1$,

$$
\begin{aligned}
& \operatorname{Span}\left\{\widehat{\Xi}_{[\mathbf{n}]}^{\Lambda} B w \mid[\mathbf{n}] \in \mathcal{N}_{\Lambda}, \Lambda \cdot \mathbf{n} \leq T, w \in \mathbb{C}\right\} \\
= & \operatorname{Span}\left\{\Xi_{\mathbf{n}} B \mid \mathbf{n}=\left(n_{1}, n_{2}\right) \in \mathbb{N}^{2}, n_{1}+\lambda n_{2} \leq T\right\} \\
\supset & \operatorname{Span}\left\{\Xi_{(0,0)} B, \Xi_{(1,0)} B, \Xi_{(0,1)} B\right\}=\mathbb{C}^{3},
\end{aligned}
$$

which shows that $\Sigma(A, B, \Lambda)$ is relatively controllable for every $T \geq 1$ when $\lambda \notin \mathbb{Q}$. However, for $\lambda=\frac{1}{2}$, one computes

$$
\widehat{\Xi}_{[\mathbf{n}]}^{\Lambda}= \begin{cases}\operatorname{Id}_{3}, & \text { if }[\mathbf{n}]=[(0,0)], \\ A_{2}, & \text { if }[\mathbf{n}]=[(0,1)], \\ 0, & \text { otherwise. }\end{cases}
$$

Thus, for every $T>0$,

$$
\operatorname{Span}\left\{\widehat{\Xi}_{[\mathbf{n}]}^{\Lambda} B w \mid[\mathbf{n}] \in \mathcal{N}_{\Lambda}, \Lambda \cdot \mathbf{n} \leq T, w \in \mathbb{C}\right\} \subset \operatorname{Span}\left\{B, A_{2} B\right\} \varsubsetneqq \mathbb{C}^{3},
$$

and hence $\Sigma(A, B, \Lambda)$ is not relatively controllable for any $T>0$ when $\lambda=\frac{1}{2}$.

Even if the converse of Theorem 4.5 does not hold in general, one can still obtain that relative controllability with a delay vector $\Lambda \in(0,+\infty)^{N}$ implies relative controllability for another delay vector $L \succcurlyeq \Lambda$ with commensurable components and sufficiently close to $\Lambda$.

Theorem 4.7. Let $A=\left(A_{1}, \ldots, A_{N}\right) \in \mathcal{M}_{d}(\mathbb{C}), B \in \mathcal{M}_{d, m}(\mathbb{C}), \Lambda=\left(\Lambda_{1}, \ldots, \Lambda_{N}\right) \in(0,+\infty)^{N}$, and $T>0$. For every $\varepsilon>0$, there exists $L=\left(L_{1}, \ldots, L_{N}\right) \in(0,+\infty)^{N}$ with commensurable components satisfying $L \succcurlyeq \Lambda$ and $1 \leq \frac{\Lambda_{j}}{L_{j}}<1+\varepsilon$ for every $j \in \llbracket 1, N \rrbracket$ such that, if $\Sigma(A, B, \Lambda)$ is relatively controllable in time $T$, then $\Sigma(A, B, L)$ is also relatively controllable in time $T$. 
Before proving Theorem 4.7, let us show the following result.

Lemma 4.8. Let $\Lambda=\left(\Lambda_{1}, \ldots, \Lambda_{N}\right) \in(0,+\infty)^{N}$ and $T>0$. For every $\varepsilon>0$, there exists $L=\left(L_{1}, \ldots\right.$, $\left.L_{N}\right) \in(0,+\infty)^{N}$ with commensurable components such that $L \succcurlyeq \Lambda, 1 \leq \frac{\Lambda_{j}}{L_{j}}<1+\varepsilon$ for every $j \in \llbracket 1, N \rrbracket$, and, for every $\mathbf{n}, \mathbf{n}^{\prime} \in \mathbb{N}^{N}$ with $\Lambda \cdot \mathbf{n} \leq T$, one has $\Lambda \cdot \mathbf{n}=\Lambda \cdot \mathbf{n}^{\prime}$ if and only if $L \cdot \mathbf{n}=L \cdot \mathbf{n}^{\prime}$.

Proof. Write $\Lambda=M \ell$, with $M=\left(m_{j k}\right)_{j \in \llbracket 1, N \rrbracket, k \in \llbracket 1, h \rrbracket} \in \mathcal{M}_{N, h}(\mathbb{N})$ for some $h \in \llbracket 1, N \rrbracket$ and $\ell=\left(\ell_{1}, \ldots\right.$, $\left.\ell_{h}\right) \in(0,+\infty)^{h}$ with rationally independent components, chosen according to Proposition 4.3. For $n \in \mathbb{N}^{*}$, we define $L^{(n)}=\left(L_{1}^{(n)}, \ldots, L_{N}^{(n)}\right) \in[0,+\infty)^{N}$ by $L^{(n)}=\frac{1}{n} M\lfloor n \ell\rfloor$, where $\lfloor n \ell\rfloor=\left(\left\lfloor n \ell_{1}\right\rfloor, \ldots\right.$, $\left.\left\lfloor n \ell_{h}\right\rfloor\right)$. We claim that $L^{(n)}$ satisfies the required properties for $n \in \mathbb{N}^{*}$ large enough.

Notice first that, if $n \geq 1 / \ell_{\min }$, then all the components of $\lfloor n \ell\rfloor$ are positive, and hence $L^{(n)} \in$ $(0,+\infty)^{N}$. Moreover, $L^{(n)} \in \mathbb{Q}^{N}$, and thus $L^{(n)}$ has commensurable components. If $\mathbf{n} \in Z(\Lambda)$, one has $\Lambda \cdot \mathbf{n}=0$, which yields $\mathbf{n}^{\mathrm{T}} M \ell=0$ and, since $\ell$ has rationally independent components and the row vector $\mathbf{n}^{\mathrm{T}} M$ has integer components, one obtains that $\mathbf{n}^{\mathrm{T}} M=0$, which implies that $L^{(n)} \cdot \mathbf{n}=$ $\frac{1}{n} \mathbf{n}^{\mathrm{T}} M\lfloor n \ell\rfloor=0$, and hence $\mathbf{n} \in Z\left(L^{(n)}\right)$, proving that $L^{(n)} \succcurlyeq \Lambda$.

For $j \in \llbracket 1, N \rrbracket$, since $n \ell_{j}-1<\left\lfloor n \ell_{j}\right\rfloor \leq n \ell_{j}$, one obtains from the definition of $L^{(n)}$ that $L_{j}^{(n)}=$ $\frac{1}{n} \sum_{k=1}^{h} m_{j k}\left\lfloor n \ell_{k}\right\rfloor \leq \Lambda_{j}$ and that $L_{j}^{(n)} \geq \Lambda_{j}-\frac{1}{n} \sum_{k=1}^{h} m_{j k} \geq \Lambda_{j}-|M|_{\infty} / n$. Hence, for $n \geq 1 / \ell_{\min }$, one has $1 \leq \frac{\Lambda_{j}}{L_{j}^{(n)}} \leq 1+\frac{|M|_{\infty}}{n L_{j}^{(n)}}$. Notice that, by construction, for every $j \in \llbracket 1, N \rrbracket$, one has $L_{j}^{(n)} \rightarrow \Lambda_{j}$ as $n \rightarrow+\infty$. Hence there exists $N_{1} \geq 1 / \ell_{\min }$ such that, for $n \geq N_{1}, L_{j}^{(n)} \geq \Lambda_{j} / 2$ for every $j \in \llbracket 1, N \rrbracket$. Thus, for $n \geq N_{1}$, one has $1 \leq \frac{\Lambda_{j}}{L_{j}^{(n)}} \leq 1+\frac{2|M|_{\infty}}{n \Lambda_{j}} \leq 1+\frac{2|M|_{\infty}}{n \Lambda_{\min }}$. Letting $N_{2} \geq N_{1}$ be such that $N_{2}>\frac{2|M|_{\infty}}{\varepsilon \Lambda_{\min }}$, one obtains that $1 \leq \frac{\Lambda_{j}}{L_{j}^{(n)}}<1+\varepsilon$ for every $j \in \llbracket 1, N \rrbracket$ and $n \geq N_{2}$.

To prove the last part of the lemma, notice that, for every $n \geq 1 / \ell_{\min }$, since $\Lambda \preccurlyeq L^{(n)}$, if $\mathbf{n}, \mathbf{n}^{\prime} \in \mathbb{N}^{N}$ are such that $\Lambda \cdot \mathbf{n}=\Lambda \cdot \mathbf{n}^{\prime}$, then $\mathbf{n}-\mathbf{n}^{\prime} \in Z(\Lambda)$ and thus $L^{(n)} \cdot \mathbf{n}=L^{(n)} \cdot \mathbf{n}^{\prime}$. Let $\mathcal{F}$ denote the finite set $\mathcal{F}=\left\{\mathbf{n} \in \mathbb{N}^{N} \mid \Lambda \cdot \mathbf{n} \leq(1+\varepsilon) T\right\}$ and define

$$
\delta=\min \left\{\left|\Lambda \cdot \mathbf{n}-\Lambda \cdot \mathbf{n}^{\prime}\right| \mid \mathbf{n}, \mathbf{n}^{\prime} \in \mathcal{F}, \Lambda \cdot \mathbf{n} \neq \Lambda \cdot \mathbf{n}^{\prime}\right\}>0 .
$$

Since $L^{(n)} \rightarrow \Lambda$ as $n \rightarrow+\infty$ and $\mathcal{F}$ is finite, there exists $N_{3} \geq N_{2}$ such that, for $n \geq N_{3}$, one has $\left|L^{(n)} \cdot \mathbf{n}-\Lambda \cdot \mathbf{n}\right|<\frac{\delta}{3}$ for every $\mathbf{n} \in \mathcal{F}$. Let $n \geq N_{3}$. Assume, to obtain a contradiction, that $\mathbf{n}, \mathbf{n}^{\prime} \in \mathbb{N}^{N}$ are such that $\Lambda \cdot \mathbf{n} \leq T, \Lambda \cdot \mathbf{n} \neq \Lambda \cdot \mathbf{n}^{\prime}$, and $L^{(n)} \cdot \mathbf{n}=L^{(n)} \cdot \mathbf{n}^{\prime}$. Then, using that $1 \leq \frac{\Lambda_{j}}{L_{j}^{(n)}}<1+\varepsilon$ for every $j \in \llbracket 1, N \rrbracket$, one computes $\Lambda \cdot \mathbf{n}^{\prime}<(1+\varepsilon) L^{(n)} \cdot \mathbf{n}^{\prime}=(1+\varepsilon) L^{(n)} \cdot \mathbf{n} \leq(1+\varepsilon) \Lambda \cdot \mathbf{n} \leq(1+\varepsilon) T$, which shows that $\mathbf{n}^{\prime} \in \mathcal{F}$. But

$$
\delta \leq\left|\Lambda \cdot \mathbf{n}-\Lambda \cdot \mathbf{n}^{\prime}\right| \leq\left|\Lambda \cdot \mathbf{n}-L^{(n)} \cdot \mathbf{n}\right|+\left|L^{(n)} \cdot \mathbf{n}-L^{(n)} \cdot \mathbf{n}^{\prime}\right|+\left|L^{(n)} \cdot \mathbf{n}^{\prime}-\Lambda \cdot \mathbf{n}^{\prime}\right|<\frac{2 \delta}{3},
$$

which is a contradiction since $\delta>0$. Hence, if $\mathbf{n}, \mathbf{n}^{\prime} \in \mathbb{N}^{N}$ are such that $\Lambda \cdot \mathbf{n} \leq T$ and $\Lambda \cdot \mathbf{n} \neq \Lambda \cdot \mathbf{n}^{\prime}$ one has $L^{(n)} \cdot \mathbf{n} \neq L^{(n)} \cdot \mathbf{n}^{\prime}$.

Proof of Theorem 4.7. Let $\varepsilon>0$ and take $L$ as in Lemma 4.8. If $\mathbf{n} \in \mathbb{N}^{N}$ is such that $\Lambda \cdot \mathbf{n} \leq T$, then $[\mathbf{n}]_{\Lambda}=[\mathbf{n}]_{L}$, since it follows from Proposition 4.4 that $[\mathbf{n}]_{\Lambda} \subset[\mathbf{n}]_{L}$ and, if $\mathbf{n}^{\prime} \in[\mathbf{n}]_{L}$, Lemma 4.8 shows that $\mathbf{n}^{\prime} \in[\mathbf{n}]_{\Lambda}$ since $\Lambda \cdot \mathbf{n} \leq T$. In particular, the only equivalence class from $\mathcal{N}_{\Lambda}$ contained in $[\mathbf{n}]_{L}$ is $[\mathbf{n}]_{\Lambda}$. Hence, Proposition 4.4 shows that, for $\mathbf{n} \in \mathbb{N}^{N}$ with $\Lambda \cdot \mathbf{n} \leq T$, one has

$$
\widehat{\Xi}_{[\mathbf{n}]}^{L}=\sum_{\substack{\tau \in \mathcal{N}_{\Lambda} \\ \tau \subset[\mathbf{n}]_{L}}} \widehat{\Xi}_{\tau}^{\Lambda}=\widehat{\Xi}_{[\mathbf{n}]}^{\Lambda},
$$


and thus

$$
\begin{aligned}
& \operatorname{Span}\left\{\widehat{\boldsymbol{\Xi}}_{[\mathbf{n}]}^{\Lambda} B w \mid[\mathbf{n}] \in \mathcal{N}_{\Lambda}, \Lambda \cdot \mathbf{n} \leq T, w \in \mathbb{C}^{m}\right\} \\
= & \operatorname{Span}\left\{\widehat{\boldsymbol{\Xi}}_{[\mathbf{n}]}^{L} B w \mid \mathbf{n} \in \mathbb{N}^{N}, \Lambda \cdot \mathbf{n} \leq T, w \in \mathbb{C}^{m}\right\} \\
\subset & \operatorname{Span}\left\{\widehat{\boldsymbol{\Xi}}_{[\mathbf{n}]}^{L} B w \mid \mathbf{n} \in \mathbb{N}^{N}, L \cdot \mathbf{n} \leq T, w \in \mathbb{C}^{m}\right\},
\end{aligned}
$$

since $L \cdot \mathbf{n} \leq \Lambda \cdot \mathbf{n}$ for every $\mathbf{n} \in \mathbb{N}^{N}$. Hence relative controllability of $\Sigma(A, B, \Lambda)$ in time $T$ implies relative controllability of $\Sigma(A, B, L)$ in time $T$.

\section{Minimal time for relative controllability}

As stated in Remark 3.3, when $N=1$ and (1.1) is written as $x(t)=A x(t-\Lambda)+B u(t)$, relative controllability in time $T$ is equivalent to Kalman condition $\operatorname{rk}\left(\begin{array}{lllll}B & A B & A^{2} B & \cdots & A^{\lfloor T / \Lambda\rfloor} B\end{array}\right)=d$. Thanks to

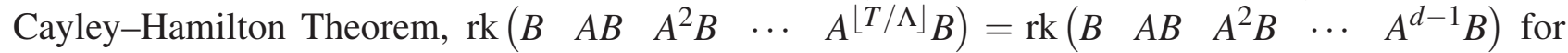
every $T \geq(d-1) \Lambda$. Hence, if the system is relatively controllable for some time $T>0$, it is also relatively controllable in time $T=(d-1) \Lambda$, which proves that its minimal controllability time $T_{\min }$ satisfies $T_{\min } \leq(d-1) \Lambda$. The uniformity of this upper bound on the matrices $A$ and $B$ is important for practical applications, since, if one is interested in finding out whether a given system is relatively controllable for some time $T>0$, it suffices to verify whether it is relatively controllable in time $T=(d-1) \Lambda$, which can be done algorithmically in a finite number of steps upper bounded by a constant independent of $A$ and $B$. The goal of this section is to generalize this upper bound on the minimal controllability time $T_{\min }$ for systems with larger $N$.

We start by considering the case of systems with commensurable delays. In this case, by considering an augmented system in higher dimension, one can characterize the relative controllability of $\Sigma(A, B, \Lambda)$ in terms of a certain output controllability of the augmented system, as shown in the next lemma.

Lemma 5.1. Let $A=\left(A_{1}, \ldots, A_{N}\right) \in \mathcal{M}_{d}(\mathbb{C})^{N}, B \in \mathcal{M}_{d, m}(\mathbb{C}), \Lambda=\left(\Lambda_{1}, \ldots, \Lambda_{N}\right) \in(0,+\infty)^{N}$, and $T>0$. Assume that $\Lambda$ has commensurable components and let $\lambda>0$ and $k_{1}, \ldots, k_{N} \in \mathbb{N}^{*}$ be such that $\left(\Lambda_{1}, \ldots, \Lambda_{N}\right)=\lambda\left(k_{1}, \ldots, k_{N}\right)$. Denote $K=\max _{j \in \llbracket 1, N \rrbracket} k_{j}$. Then $\Sigma(A, B, \Lambda)$ is relatively controllable in time $T>0$ if and only if, for every $X_{0}:[-\lambda, 0) \rightarrow \mathbb{C}^{K d}$ and $x_{1} \in \mathbb{C}^{d}$, there exists $u:[0, T] \rightarrow \mathbb{C}^{m}$ such that the unique solution $X:[-\lambda, T] \rightarrow \mathbb{C}^{K d}$ of

$$
\begin{cases}X(t)=\widehat{A} X(t-\lambda)+\widehat{B} u(t), & t \in[0, T], \\ X(t)=X_{0}(t), & t \in[-\lambda, 0),\end{cases}
$$

satisfies $\widehat{C} X(T)=x_{1}$, where the matrices $\widehat{A} \in \mathcal{M}_{K d}(\mathbb{C}), \widehat{B} \in \mathcal{M}_{K d, m}(\mathbb{C})$, and $\widehat{C} \in \mathcal{M}_{d, K d}(\mathbb{C})$ are given by

$$
\begin{aligned}
& \widehat{A}=\left(\begin{array}{ccccc}
\widehat{A}_{1} & \widehat{A}_{2} & \widehat{A}_{3} & \cdots & \widehat{A}_{K} \\
\operatorname{Id}_{d} & 0 & 0 & \cdots & 0 \\
0 & \operatorname{Id}_{d} & 0 & \cdots & 0 \\
\vdots & \vdots & \ddots & \ddots & \vdots \\
0 & 0 & \cdots & \operatorname{Id}_{d} & 0
\end{array}\right) \in \mathcal{M}_{K d}(\mathbb{C}), \quad \widehat{B}=\left(\begin{array}{c}
B \\
0 \\
0 \\
\vdots \\
0
\end{array}\right) \in \mathcal{M}_{K d, m}(\mathbb{C}), \\
& \widehat{C}=\left(\begin{array}{ccccc}
\operatorname{Id}_{d} & 0 & 0 & \cdots & 0
\end{array}\right) \in \mathcal{M}_{d, K d}(\mathbb{C}), \widehat{A}_{k}=\sum_{\substack{j=1 \\
k_{j}=k}}^{N} A_{j} \quad \text { for } k \in \llbracket 1, K \rrbracket,
\end{aligned}
$$


Proof. It is immediate to verify that $x:\left[-\Lambda_{\max }, T\right] \rightarrow \mathbb{C}^{d}$ is the solution of $\Sigma(A, B, \Lambda)$ with initial condition $x_{0}:\left[-\Lambda_{\max }, 0\right) \rightarrow \mathbb{C}^{d}$ and control $u:[0, T] \rightarrow \mathbb{C}^{m}$ if and only if the function $X:[-\lambda, T] \rightarrow$ $\mathbb{C}^{K d}$ defined by

$$
X(t)=\left(\begin{array}{c}
x(t) \\
x(t-\lambda) \\
x(t-2 \lambda) \\
\vdots \\
x(t-(K-1) \lambda)
\end{array}\right)
$$

is the solution of (5.1) with control $u$ and with initial condition $X_{0}:[\lambda, 0) \rightarrow \mathbb{C}^{K d}$ given by

$$
X_{0}(t)=\left(\begin{array}{c}
x_{0}(t) \\
x_{0}(t-\lambda) \\
x_{0}(t-2 \lambda) \\
\vdots \\
x_{0}(t-(K-1) \lambda)
\end{array}\right) .
$$

Since $\widehat{C} X(t)=x(t)$ for every $t \in[-\lambda, T]$, the statement of the lemma follows immediately from Theorem 3.1.

Since (5.1) is a controlled difference equation with a single delay, we use Lemma 5.1 to characterize the relative controllability of $\Sigma(A, B, \Lambda)$ in terms of a Kalman rank condition.

Corollary 5.2. Let $A=\left(A_{1}, \ldots, A_{N}\right) \in \mathcal{M}_{d}(\mathbb{C})^{N}, B \in \mathcal{M}_{d, m}(\mathbb{C}), \Lambda=\left(\Lambda_{1}, \ldots, \Lambda_{N}\right) \in(0,+\infty)^{N}$, and $T>0$. Assume that $\Lambda$ has commensurable components. Then $\Sigma(A, B, \Lambda)$ is relatively controllable in time $T$ if and only if

$$
\operatorname{rk}\left(\widehat{C} \widehat{B} \quad \widehat{C} \widehat{A} \widehat{B} \quad \widehat{C} \widehat{A}^{2} \widehat{B} \quad \cdots \quad \widehat{C} \widehat{A}^{\lfloor T / \lambda\rfloor} \widehat{B}\right)=d,
$$

where $\widehat{A}, \widehat{B}, \widehat{C}$, and $\lambda$ are as in the statement of Lemma 5.1.

Proof. Notice that, by Proposition 2.7, the solution $X:[-\lambda, T] \rightarrow \mathbb{C}^{K d}$ of (5.1) with initial condition $X_{0}:[-\lambda, 0) \rightarrow \mathbb{C}^{K d}$ and control $u:[0, T] \rightarrow \mathbb{C}^{m}$ is given by

$$
X(t)=\widehat{A}^{1+\lfloor t / \lambda\rfloor} X_{0}\left(t-\left(1+\left\lfloor\frac{t}{\lambda}\right\rfloor\right) \lambda\right)+\sum_{n=0}^{\lfloor t / \lambda\rfloor} \widehat{A}^{n} \widehat{B} u(t-n \lambda) .
$$

Hence

$$
\widehat{C} X(T)=\widehat{C} \widehat{A}^{1+\lfloor T / \lambda\rfloor} X_{0}\left(T-\left(1+\left\lfloor\frac{T}{\lambda}\right\rfloor\right) \lambda\right)+\sum_{n=0}^{\lfloor T / \lambda\rfloor} \widehat{C} \widehat{A}^{n} \widehat{B} u(T-n \lambda) .
$$

If $\Sigma(A, B, \Lambda)$ is relatively controllable in time $T$, then, by Lemma 5.1, taking $X_{0}=0$, one obtains that, for every $x_{1} \in \mathbb{C}^{d}$, there exists $u:[0, T] \rightarrow \mathbb{C}^{m}$ such that $\sum_{n=0}^{\lfloor T / \lambda\rfloor} \widehat{C} \widehat{A^{n}} \widehat{B} u(T-n \lambda)=x_{1}$, which shows that (5.3) holds. Conversely, if (5.3) holds, it follows that the matrix $\left(\begin{array}{llll}\widehat{C} \widehat{B} & \widehat{C} \widehat{A} \widehat{B} & \cdots & \widehat{C} \widehat{A} \\ \mid T / \lambda\rfloor & \widehat{B}\end{array}\right)$ admits a right inverse $M \in \mathcal{M}_{(\lfloor T / \lambda\rfloor+1) m, d}(\mathbb{C})$. For $X_{0}:[-\lambda, 0) \rightarrow \mathbb{C}^{K d}$ and $x_{1} \in \mathbb{C}^{d}$, let $U=\left(U_{j}\right)_{j=0}^{\lfloor T / \lambda\rfloor}$ $\in \mathbb{C}^{(\lfloor T / \lambda\rfloor+1) m}$ be given by

$$
U=\left(\begin{array}{c}
U_{0} \\
\vdots \\
U_{\lfloor T / \lambda\rfloor}
\end{array}\right)=M\left[x_{1}-\widehat{C} \widehat{A}^{1+\lfloor T / \lambda\rfloor} X_{0}\left(T-\left(1+\left\lfloor\frac{T}{\lambda}\right\rfloor\right) \lambda\right)\right]
$$

and take $u:[0, T] \rightarrow \mathbb{C}^{m}$ satisfying $u(T-n \lambda)=U_{n}$ for every $n \in \llbracket 0,\lfloor T / \lambda\rfloor \rrbracket$. It follows immediately from (5.4) that the solution of (5.1) with initial condition $X_{0}$ and control $u$ satisfies $\widehat{C} X(T)=x_{1}$, and hence, by Lemma 5.1, $\Sigma(A, B, \Lambda)$ is relatively controllable in time $T$. 
Thanks to Cayley-Hamiltion Theorem, Corollary 5.2 allows one to obtain an upper bound on the minimal controllability time for $\Sigma(A, B, \Lambda)$ with commensurable delays.

Lemma 5.3. Let $A=\left(A_{1}, \ldots, A_{N}\right) \in \mathcal{M}_{d}(\mathbb{C})^{N}, B \in \mathcal{M}_{d, m}(\mathbb{C})$, and $\Lambda=\left(\Lambda_{1}, \ldots, \Lambda_{N}\right) \in(0,+\infty)^{N}$. Assume that $\Lambda$ has commensurable components. If there exists $T>0$ such that $\Sigma(A, B, \Lambda)$ is relatively controllable in time $T$, then its minimal controllability time $T_{\min }$ satisfies $T_{\min } \leq(d-1) \Lambda_{\max }$.

Proof. For $j \in \llbracket 1, K \rrbracket$, set

$$
\widehat{C}_{j}=\left(\begin{array}{lll}
0_{d,(j-1) d} & \left.\operatorname{Id}_{d} \quad 0_{d,(K-j) d}\right) \in \mathcal{M}_{d, K d}(\mathbb{C}) .
\end{array}\right.
$$

In particular, $\widehat{C}_{1}=\widehat{C}$. For every $j \in \llbracket 2, K \rrbracket$, one has $\widehat{C}_{j} \widehat{A}=\widehat{C}_{j-1}$, and thus $\widehat{C}=\widehat{C}_{K} \widehat{A}^{K-1}$. Hence, for every $k \in \mathbb{N}$, one has

$$
\left(\begin{array}{llllll}
\widehat{C} \widehat{B} & \widehat{C} \widehat{A} \widehat{B} & \widehat{C} \widehat{A}^{2} \widehat{B} & \cdots & \widehat{C} \widehat{A}^{k} \widehat{B}
\end{array}\right)=\left(\begin{array}{lllll}
\widehat{C}_{K} \widehat{A}^{K-1} \widehat{B} & \widehat{C}_{K} \widehat{A}^{K} \widehat{B} & \widehat{C}_{K} \widehat{A}^{K+1} \widehat{B} & \cdots & \widehat{C}_{K} \widehat{A}^{K+k-1} \widehat{B}
\end{array}\right) .
$$

Moreover, since $\widehat{C}_{K} \widehat{A}^{j}=\widehat{C}_{K-j}$ for every $j \in \llbracket 0, K-1 \rrbracket$, one computes, for $j \in \llbracket 0, K-2 \rrbracket, \widehat{C}_{K} \widehat{A}^{j} \widehat{B}=$ $\widehat{C}_{K-j} \widehat{B}=0$, which shows that

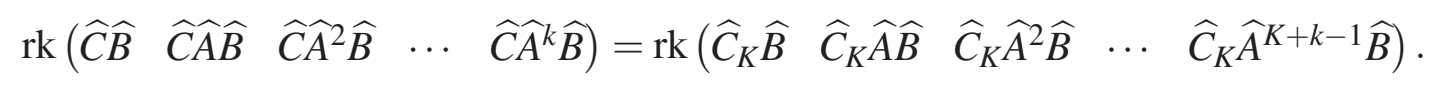

Let $T>0$ be such that $\Sigma(A, B, \Lambda)$ is relatively controllable in time $T$. If $T \leq(d-1) \Lambda_{\max }$, one has immediately that $T_{\min } \leq(d-1) \Lambda_{\max }$. If $T>(d-1) \Lambda_{\max }$, one has, by Corollary 5.2 and (5.5), that

$$
\operatorname{rk}\left(\widehat{C}_{K} \widehat{B} \quad \widehat{C}_{K} \widehat{A} \widehat{B} \quad \widehat{C}_{K} \widehat{A}^{2} \widehat{B} \quad \cdots \quad \widehat{C}_{K} \widehat{A}^{K+\lfloor T / \lambda\rfloor-1} \widehat{B}\right)=d .
$$

By Cayley-Hamilton Theorem, since $\widehat{A} \in \mathcal{M}_{K d}(\mathbb{C})$, this implies that

$$
\begin{aligned}
& d=\operatorname{rk}\left(\begin{array}{llllll}
\widehat{C}_{K} \widehat{B} & \widehat{C}_{K} \widehat{A} \widehat{B} & \widehat{C}_{K} \widehat{A}^{2} \widehat{B} & \cdots & \left.\widehat{C}_{K} \widehat{A}^{K+\lfloor T / \lambda\rfloor-1} \widehat{B}\right)
\end{array}\right. \\
& =\operatorname{rk}\left(\begin{array}{lllll}
\widehat{C}_{K} \widehat{B} & \widehat{C}_{K} \widehat{A} \widehat{B} & \widehat{C}_{K} \widehat{A}^{2} \widehat{B} & \cdots & \widehat{C}_{K} \widehat{A}^{K d-1} \widehat{B}
\end{array}\right)
\end{aligned}
$$

since $K+\lfloor T / \lambda\rfloor-1 \geq K d-1$. Hence, by Corollary 5.2 and (5.5), it follows that $\Sigma(A, B, \Lambda)$ is relatively controllable in time $T=K(d-1) \lambda=(d-1) \Lambda_{\max }$, which yields $T_{\min } \leq(d-1) \Lambda_{\max }$.

Now that Lemma 5.3 has established a uniform upper bound on the minimal controllability time for $\Sigma(A, B, \Lambda)$ with commensurate delays, one can use Theorems 4.5 and 4.7 in order to deduce a uniform upper bound for all delay vectors $\Lambda \in(0,+\infty)^{N}$.

Theorem 5.4. Let $A=\left(A_{1}, \ldots, A_{N}\right) \in \mathcal{M}_{d}(\mathbb{C})^{N}, B \in \mathcal{M}_{d, m}(\mathbb{C})$, and $\Lambda=\left(\Lambda_{1}, \ldots, \Lambda_{N}\right) \in(0,+\infty)^{N}$. If there exists $T>0$ such that $\Sigma(A, B, \Lambda)$ is relatively controllable in time $T$, then its minimal controllability time $T_{\min }$ satisfies $T_{\min } \leq(d-1) \Lambda_{\max }$.

Proof. Let $\varepsilon>0$ and choose $L \in(0,+\infty)^{N}$ according to Theorem 4.7. Then $\Sigma(A, B, L)$ is relatively controllable in time $T$. Thanks to Lemma 5.3, the minimal controllability time $T_{\min }^{(L)}$ for $\Sigma(A, B, L)$ satisfies $T_{\min }^{(L)} \leq(d-1) L_{\max }$, and, in particular, $\Sigma(A, B, L)$ is relatively controllable in time $(d-1) L_{\max }$. Hence, by Theorem $4.5, \Sigma(A, B, \Lambda)$ is relatively controllable in time $(1+\varepsilon)(d-1) L_{\max }$, which proves that the minimal controllability time $T_{\min }$ for $\Sigma(A, B, \Lambda)$ satisfies $T_{\min } \leq(1+\varepsilon)(d-1) L_{\max } \leq(1+$ $\varepsilon)(d-1) \Lambda_{\max }$. Since $\varepsilon>0$ is arbitrary, one concludes that $T_{\min } \leq(d-1) \Lambda_{\max }$. 
Remark 5.5. The statements and proofs of the results from this section and the previous one can be slightly modified to show that, for every $A=\left(A_{1}, \ldots, A_{N}\right) \in \mathcal{M}_{d}(\mathbb{C})^{N}, B \in \mathcal{M}_{d, m}(\mathbb{C}), \Lambda=\left(\Lambda_{1}, \ldots, \Lambda_{N}\right)$ $\in(0,+\infty)^{N}$, and $T \geq(d-1) \Lambda_{\max }$, one has

$$
\begin{aligned}
& \operatorname{Span}\left\{\widehat{\Xi}_{[\mathbf{n}]}^{\Lambda} B w \mid[\mathbf{n}] \in \mathcal{N}_{\Lambda}, \Lambda \cdot \mathbf{n} \leq T, w \in \mathbb{C}^{m}\right\} \\
= & \operatorname{Span}\left\{\widehat{\Xi}_{[\mathbf{n}]}^{\Lambda} B w \mid[\mathbf{n}] \in \mathcal{N}_{\Lambda}, \Lambda \cdot \mathbf{n} \leq(d-1) \Lambda_{\max }, w \in \mathbb{C}^{m}\right\} .
\end{aligned}
$$

The set $\mathrm{V}=\operatorname{Span}\left\{\widehat{\mathbb{\Xi}}_{[\mathbf{n}]}^{\Lambda} B w \mid[\mathbf{n}] \in \mathcal{N}_{\Lambda}, \Lambda \cdot \mathbf{n} \leq(d-1) \Lambda_{\max }, w \in \mathbb{C}^{m}\right\}$ is the set of all states $x_{1} \in \mathbb{C}^{d}$ that can be reached by the system $\Sigma(A, B, \Lambda)$ after time $T \geq(d-1) \Lambda_{\max }$ starting from a zero initial condition.

When $N=1$ and the controlled difference equation (1.1) becomes $x(t)=A x(t-\Lambda)+B u(t)$ with $A=A_{1}$ and $\Lambda=\Lambda_{1}$, Kalman decomposition (see, e.g., [33, Lemma 3.3.3]) states that there exists an invertible matrix $P \in \mathcal{M}_{d}(\mathbb{C})$ such that

$$
P A P^{-1}=\left(\begin{array}{cc}
A_{11} & A_{12} \\
0 & A_{22}
\end{array}\right), \quad P B=\left(\begin{array}{c}
B_{1} \\
0
\end{array}\right)
$$

with $A_{11} \in \mathcal{M}_{r}(\mathbb{C}), A_{22} \in \mathcal{M}_{d-r}(\mathbb{C}), B_{1} \in \mathcal{M}_{r, m}(\mathbb{C})$, where $r=\operatorname{dim} \mathrm{V}$, the pair $\left(A_{11}, B_{1}\right)$ is controllable, and $P \bigvee=\mathbb{C}^{r} \times\{0\}^{d-r}=\operatorname{Span}\left\{e_{1}, \ldots, e_{r}\right\}$.

Such decomposition does not hold for larger $N$ in general, i.e., one cannot find in general, for $A=\left(A_{1}, \ldots, A_{N}\right) \in \mathcal{M}_{d}(\mathbb{C})^{N}, B \in \mathcal{M}_{d, m}(\mathbb{C})$, and $\Lambda \in(0,+\infty)^{N}$ for which $\Sigma(A, B, \Lambda)$ is not relatively controllable in any time $T>0$, a matrix $P \in \mathcal{M}_{d}(\mathbb{C})$ for which one would have, for every $j \in \llbracket 1, N \rrbracket$,

$$
P A_{j} P^{-1}=\left(\begin{array}{cc}
A_{11}^{(j)} & A_{12}^{(j)} \\
0 & A_{22}^{(j)}
\end{array}\right), \quad P B=\left(\begin{array}{c}
B_{1} \\
0
\end{array}\right)
$$

with $A_{11}^{(j)} \in \mathcal{M}_{r}(\mathbb{C}), A_{22}^{(j)} \in \mathcal{M}_{d-r}(\mathbb{C}), B_{1} \in \mathcal{M}_{r, m}(\mathbb{C})$, with $r \in \llbracket 0, d-1 \rrbracket$ and such that $\Sigma\left(A_{11}^{(1)}, \ldots, A_{11}^{(N)}\right.$, $\left.B_{1}, \Lambda\right)$ is relatively controllable in time $T \geq(r-1) \Lambda_{\max }$. Indeed, consider the case $N=2, d=4$, $m=1, \Lambda=(1, \ell)$ for some $\ell \in\left(\frac{3}{4}, 1\right)$, and

$$
A_{1}=\left(\begin{array}{cccc}
0 & 1 & 0 & 0 \\
2 & 0 & 0 & 0 \\
0 & 0 & 0 & 1 \\
-3 & \sqrt{2} & 0 & 0
\end{array}\right), \quad A_{2}=\left(\begin{array}{cccc}
\frac{1}{2} & 0 & -1 & 0 \\
0 & 1 & 0 & 1 \\
0 & 0 & 1 & 0 \\
\sqrt{3} & 0 & 0 & 2
\end{array}\right), \quad B=\left(\begin{array}{l}
0 \\
0 \\
0 \\
1
\end{array}\right)
$$

Notice that

$$
\begin{aligned}
& \operatorname{Span}\left\{\Xi_{\mathbf{n}} B \mid \mathbf{n}=\left(n_{1}, n_{2}\right) \in \mathbb{N}^{2}, n_{1}+\ell n_{2} \leq 3\right\} \\
= & \operatorname{Span}\left\{\Xi_{(0,0)} B, \Xi_{(0,1)} B, \Xi_{(0,2)} B, \Xi_{(0,3)} B, \Xi_{(1,0)} B, \Xi_{(1,1)} B, \Xi_{(1,2)} B, \Xi_{(2,0)} B, \Xi_{(2,1)} B, \Xi_{(3,0)} B\right\} \\
= & \operatorname{Span}\left\{\left(\begin{array}{l}
0 \\
0 \\
0 \\
1
\end{array}\right),\left(\begin{array}{l}
0 \\
1 \\
0 \\
2
\end{array}\right),\left(\begin{array}{l}
0 \\
3 \\
0 \\
4
\end{array}\right),\left(\begin{array}{l}
0 \\
7 \\
0 \\
8
\end{array}\right),\left(\begin{array}{l}
0 \\
0 \\
1 \\
0
\end{array}\right),\left(\begin{array}{c}
0 \\
0 \\
3 \\
\sqrt{2}
\end{array}\right),\left(\begin{array}{c}
0 \\
\sqrt{2} \\
7 \\
5 \sqrt{2}
\end{array}\right),\left(\begin{array}{c}
0 \\
0 \\
\sqrt{2} \\
0
\end{array}\right)\right\} \\
= & \{0\} \times \mathbb{C}^{3},
\end{aligned}
$$

and thus, by the definition of relative controllability and Theorem 4.5 , one obtains that $\Sigma(A, B, \Lambda)$ is not relatively controllable in any time $T>0$. We claim that this system cannot be decomposed under the form (5.6). If it were the case, one immediately verifies from (5.6) that the vector space 
$\mathrm{V}=P^{-1}\left(\mathbb{C}^{r} \times\{0\}^{4-r}\right)$ would contain $B$ and be invariant under left multiplication by $A_{1}$ and $A_{2}$. Such invariance implies in particular that $\Xi_{\mathbf{n}} B \in \mathrm{V}$ for every $\mathbf{n} \in \mathbb{N}^{2}$, and thus $\{0\} \times \mathbb{C}^{3} \subset \mathrm{V}$. Such invariance then also implies that

$$
\vee \ni A_{1}\left(\begin{array}{l}
0 \\
1 \\
0 \\
0
\end{array}\right)=\left(\begin{array}{c}
1 \\
0 \\
0 \\
\sqrt{2}
\end{array}\right)
$$

which shows that $\mathrm{V}=\mathbb{C}^{4}$, contradicting the fact that $\mathrm{V}=P^{-1}\left(\mathbb{C}^{r} \times\{0\}^{4-r}\right)$ for an invertible $P \in$ $\mathcal{M}_{4}(\mathbb{C})$ and $r \in \llbracket 0,3 \rrbracket$. Hence $\Sigma(A, B, \Lambda)$ cannot be put under the form (5.6).

Example 5.6. Let $A \in \mathcal{M}_{d}(\mathbb{C}), B \in \mathcal{M}_{d, m}(\mathbb{C}), k \in \mathbb{N}^{*}$ and consider the difference equation

$$
x(t)=x(t-1)+A x(t-k)+B u(t),
$$

which we write under the form (1.1) by setting $A_{1}=\operatorname{Id}_{d}, A_{2}=A, \Lambda_{1}=1$, and $\Lambda_{2}=k$. Notice that, by taking only integer times, (5.7) can be seen as an implicit Euler discretization of the continuous-time delayed control system $\dot{x}(t)=A_{0} x(t-\tau)+B_{0} u(t)$ with time step $h=\frac{\tau}{k}$ and $A=h A_{0}, B=h B_{0}$, and is similar to the system (1.3) obtained by an explicit Euler discretization.

One easily verifies using (2.2) that the matrix coefficients $\Xi_{\mathbf{n}}$ associated with (5.7) are given for $\mathbf{n}=\left(n_{1}, n_{2}\right) \in \mathbb{N}^{2}$ by

$$
\Xi_{\mathbf{n}}=\left(\begin{array}{c}
n_{1}+n_{2} \\
n_{1}
\end{array}\right) A^{n_{2}}
$$

and one then obtains from Definition 2.8 that

$$
\widehat{\Xi}_{[\mathbf{n}]}^{\Lambda}=\sum_{j=0}^{\left\lfloor\frac{n_{1}}{k}+n_{2}\right\rfloor}\left(\begin{array}{c}
n_{1}+k n_{2}-j(k-1) \\
j
\end{array}\right) A^{j} .
$$

Hence $\widehat{\Xi}_{[\mathbf{n}]}^{\Lambda}$ coincides with the discrete delayed matrix exponential $e_{k-1}^{A\left(n_{1}+1+k\left(n_{2}-1\right)\right)}$ introduced in [12]. It follows from (5.8) that

$$
\operatorname{Span}\left\{\widehat{\Xi}_{[\mathbf{n}]}^{\Lambda} B w \mid[\mathbf{n}] \in \mathcal{N}_{\Lambda}, \Lambda \cdot \mathbf{n} \leq T, w \in \mathbb{C}^{m}\right\}=\operatorname{Ran}\left(\begin{array}{lllll}
B & A B & A^{2} B \quad \cdots \quad A^{\lfloor T / k\rfloor} B
\end{array}\right),
$$

and thus, by Theorem 3.1, (5.7) is relatively controllable in time $T$ if and only if

$$
\operatorname{rk}\left(B \quad A B \quad A^{2} B \quad \cdots \quad A^{\lfloor T / k\rfloor} B\right)=d,
$$

its minimal controllability time $T_{\min }$ satisfying $T_{\min } \leq k(d-1)$ thanks to Theorem 5.4 (this is also an immediate consequence of (5.9) and Cayley-Hamilton theorem in this case). In particular, in the single-input case $m=1$, the minimal controllability time is $T_{\min }=k(d-1)$, since the rank of the matrix in (5.9) is upper bounded by $d-1$ when $T<k(d-1)$. Notice that this is very similar to the relative controllability criterion for (1.3) proved in [13, Theorem 3.1].

Theorem 5.4 shows that, given $A=\left(A_{1}, \ldots, A_{N}\right) \in \mathcal{M}_{d}(\mathbb{C})^{N}, B \in \mathcal{M}_{d, m}(\mathbb{C})$, and $\Lambda \in(0,+\infty)^{N}$, if one wants to check whether $\Sigma(A, B, \Lambda)$ is relatively controllable in some time $T>0$, it suffices to verify whether it is relatively controllable in time $(d-1) \Lambda_{\max }$, i.e., if

$$
\operatorname{Span}\left\{\widehat{\Xi}_{[\mathbf{n}]}^{\Lambda} B w \mid[\mathbf{n}] \in \mathcal{N}_{\Lambda}, \Lambda \cdot \mathbf{n} \leq(d-1) \Lambda_{\max }, w \in \mathbb{C}^{m}\right\}=\mathbb{C}^{d}
$$

or, equivalently, if

$$
\operatorname{Span}\left\{\widehat{\mathbb{\Xi}}_{[\mathbf{n}]}^{\Lambda} B e_{j} \mid[\mathbf{n}] \in \mathcal{N}_{\Lambda}, \Lambda \cdot \mathbf{n} \leq(d-1) \Lambda_{\max }, j \in \llbracket 1, m \rrbracket\right\}=\mathbb{C}^{d},
$$


where $e_{1}, \ldots, e_{m}$ is the canonical basis of $\mathbb{C}^{m}$. The set whose span is evaluated in the left-hand side of (5.10) is finite, its cardinality being upper bounded by $m \#\left\{\left.\mathbf{n} \in \mathbb{N}^{N}|| \mathbf{n}\right|_{1} \leq(d-1) \Lambda_{\max } / \Lambda_{\min }\right\}$, which is large when $\Lambda_{\max } / \Lambda_{\min }$ is large. The next results provides a way of improving such upper bound, and hence reducing the number of elements to be evaluated in order to study the relative controllability of $\Sigma(A, B, \Lambda)$.

Theorem 5.7. Let $A=\left(A_{1}, \ldots, A_{N}\right) \in \mathcal{M}_{d}(\mathbb{C})^{N}, B \in \mathcal{M}_{d, m}(\mathbb{C})$, and $\Lambda, L \in(0,+\infty)^{N}$ with $\Lambda \preccurlyeq L$. Then $\Sigma(A, B, \Lambda)$ is relatively controllable in some time $T>0$ if and only if

$$
\operatorname{Span}\left\{\widehat{\mathbb{\Xi}}_{[\mathbf{n}]}^{\Lambda} B e_{j} \mid[\mathbf{n}] \in \mathcal{N}_{\Lambda}, L \cdot \mathbf{n} \leq(d-1) L_{\max }, j \in \llbracket 1, m \rrbracket\right\}=\mathbb{C}^{d} .
$$

Proof. If (5.11) is satisfied, then, since $\Lambda \cdot \mathbf{n} \leq \frac{\Lambda_{\max }}{L_{\min }} L \cdot \mathbf{n}$ for every $\mathbf{n} \in \mathbb{N}^{N}$, one obtains that

$$
\begin{aligned}
\mathbb{C}^{d} & =\operatorname{Span}\left\{\widehat{\Xi}_{[\mathbf{n}]}^{\Lambda} B e_{j} \mid[\mathbf{n}] \in \mathcal{N}_{\Lambda}, L \cdot \mathbf{n} \leq(d-1) L_{\max }, j \in \llbracket 1, m \rrbracket\right\} \\
& \subset \operatorname{Span}\left\{\widehat{\Xi}_{[\mathbf{n}]}^{\Lambda} B e_{j} \mid[\mathbf{n}] \in \mathcal{N}_{\Lambda}, \Lambda \cdot \mathbf{n} \leq(d-1) \Lambda_{\max } \frac{L_{\max }}{L_{\min }}, j \in \llbracket 1, m \rrbracket\right\}
\end{aligned}
$$

which proves that $\Sigma(A, B, \Lambda)$ is relatively controllable in time $T=(d-1) \Lambda_{\max } \frac{L_{\max }}{L_{\min }}$, and thus also in time $T=(d-1) \Lambda_{\max }$ thanks to Theorem 5.4.

Let $\varepsilon>0$. Write $\Lambda=M \ell$, with $M \in \mathcal{M}_{N, h}(\mathbb{N})$ for some $h \in \llbracket 1, N \rrbracket$ and $\ell=\left(\ell_{1}, \ldots, \ell_{h}\right) \in(0,+\infty)^{h}$ with rationally independent components, chosen according to Proposition 4.3. Since $\Lambda \preccurlyeq L$, it follows from Proposition 4.3 that $L \in \operatorname{Ran} M$, and thus there exists $r \in \mathbb{R}^{h}$ such that $L=M r$. Take $r_{\varepsilon} \in$ $\mathbb{R}^{h}$ with rationally independent components satisfying $\left|r-r_{\varepsilon}\right|_{\infty}<\varepsilon /|M|_{\infty}$, and set $L_{\varepsilon}=M r_{\varepsilon}$. Then $\left|L-L_{\varepsilon}\right|_{\infty}<\varepsilon$ and, in particular, $L_{\varepsilon} \in(0,+\infty)^{N}$ for $\varepsilon$ small enough. Notice that $L_{\varepsilon} \approx \Lambda$, since $\Lambda \preccurlyeq L_{\varepsilon}$ by construction and, if $\mathbf{n} \in \mathbb{N}^{N}$ is such that $L_{\varepsilon} \cdot \mathbf{n}=0$, then $\mathbf{n}^{\mathrm{T}} M r_{\varepsilon}=0$, which implies, from the fact that $r_{\varepsilon}$ has rationally independent components and that $\mathbf{n}^{\mathrm{T}} M$ is a row vector of integers, that $\mathbf{n}^{\mathrm{T}} M=0$, yielding $\Lambda \cdot \mathbf{n}=\mathbf{n}^{\mathrm{T}} M \ell=0$, and thus $L_{\varepsilon} \preccurlyeq \Lambda$. Since $\Lambda \approx L_{\varepsilon}$, it follows from Theorem 4.5 that $\Sigma(A, B, \Lambda)$ is relatively controllable in some time $T>0$ if and only if $\Sigma\left(A, B, L_{\varepsilon}\right)$ is relatively controllable in some time, i.e.,

$$
\operatorname{Span}\left\{\widehat{\Xi}_{[\mathbf{n}]}^{L_{\varepsilon}} B e_{j} \mid[\mathbf{n}] \in \mathcal{N}_{L_{\varepsilon}}, L_{\varepsilon} \cdot \mathbf{n} \leq(d-1) L_{\varepsilon \max }, j \in \llbracket 1, m \rrbracket\right\}=\mathbb{C}^{d} .
$$

By Proposition 4.4, this is equivalent to

$$
\operatorname{Span}\left\{\widehat{\Xi}_{[\mathbf{n}]}^{\Lambda} B e_{j} \mid[\mathbf{n}] \in \mathcal{N}_{\Lambda}, L_{\varepsilon} \cdot \mathbf{n} \leq(d-1) L_{\varepsilon \max }, j \in \llbracket 1, m \rrbracket\right\}=\mathbb{C}^{d} .
$$

Notice that, if $\varepsilon$ is small enough, then, for every $\mathbf{n} \in \mathbb{N}^{N}, L_{\varepsilon} \cdot \mathbf{n} \leq(d-1) L_{\varepsilon} \max$ implies $L \cdot \mathbf{n} \leq$ $(d-1) L_{\max }$. Indeed, assume that, for every $\varepsilon>0$, there exists $\mathbf{n}_{\varepsilon} \in \mathbb{N}^{N}$ such that $L_{\varepsilon} \cdot \mathbf{n}_{\varepsilon} \leq(d-1) L_{\varepsilon} \max$ and $L \cdot \mathbf{n}_{\varepsilon}>(d-1) L_{\max }$. Then $(d-1) L_{\max }<L \cdot \mathbf{n}_{\varepsilon} \leq(d-1) L_{\varepsilon} \max +\left(L-L_{\varepsilon}\right) \cdot \mathbf{n}_{\varepsilon}$, which implies that $(d-1) L_{\max }<L \cdot \mathbf{n}_{\varepsilon} \leq(d-1) L_{\max }+\varepsilon\left(d-1+\left|\mathbf{n}_{\varepsilon}\right|_{1}\right)$ and so

$$
(d-1) L_{\max }<L \cdot \mathbf{n}_{\varepsilon} \leq(d-1) L_{\max }+\varepsilon(d-1)\left(1+\frac{L_{\varepsilon \max }}{L_{\varepsilon \min }}\right)
$$

Since the set $\left\{L \cdot \mathbf{n} \mid \mathbf{n} \in \mathbb{N}^{N}\right\} \cap[0, \tau]$ is finite for every $\tau \geq 0$, one obtains that, for every $K \geq 0$, the set $\left\{\mathbf{n} \in \mathbb{N}^{N} \mid K<L \cdot \mathbf{n} \leq K+\delta\right\}$ is empty if $\delta>0$ is small enough. Hence, since $L_{\varepsilon} \max / L_{\varepsilon} \min \rightarrow$ $L_{\max } / L_{\min }$ as $\varepsilon \rightarrow 0$, one obtains that, for $\varepsilon>0$ small enough, (5.13) cannot be satisfied, which proves that $L_{\varepsilon} \cdot \mathbf{n} \leq(d-1) L_{\varepsilon} \max$ implies $L \cdot \mathbf{n} \leq(d-1) L_{\max }$ for $\varepsilon>0$ small enough.

If $\Sigma(A, B, \Lambda)$ is relatively controllable in some time, then (5.12) is satisfied. Hence, for $\varepsilon>0$ small enough,

$$
\mathbb{C}^{d}=\operatorname{Span}\left\{\widehat{\Xi}_{[\mathbf{n}]}^{\Lambda} B e_{j} \mid[\mathbf{n}] \in \mathcal{N}_{\Lambda}, L_{\varepsilon} \cdot \mathbf{n} \leq(d-1) L_{\varepsilon \max }, j \in \llbracket 1, m \rrbracket\right\}
$$




$$
\subset \operatorname{Span}\left\{\widehat{\mathbb{\Xi}}_{[\mathbf{n}]}^{\Lambda} B e_{j} \mid[\mathbf{n}] \in \mathcal{N}_{\Lambda}, L \cdot \mathbf{n} \leq(d-1) L_{\max }, j \in \llbracket 1, m \rrbracket\right\},
$$

which proves (5.11).

Notice that the set whose span is evaluated on the left-hand side of (5.11) has at most $m \#\{\mathbf{n} \in$ $\left.\left.\mathbb{N}^{N}|| \mathbf{n}\right|_{1} \leq(d-1) L_{\max } / L_{\min }\right\}$ elements, which is an improvement with respect to the upper bound obtained previously for the set whose span is evaluated on the left-hand side of (5.10) as soon as $L_{\max } / L_{\min }<\Lambda_{\max } / \Lambda_{\min }$. Hence Theorem 5.7 allows one to algorithmically check whether $\Sigma(A, B, \Lambda)$ is relatively controllable in less steps than by using (5.10). In particular, since we have $\Lambda \preccurlyeq(1,1, \ldots, 1)$ for every $\Lambda \in(0,+\infty)^{N}$ with rationally independent components, one obtains the following improvement of (5.10) in this case.

Corollary 5.8. Let $A=\left(A_{1}, \ldots, A_{N}\right) \in \mathcal{M}_{d}(\mathbb{C})^{N}, B \in \mathcal{M}_{d, m}(\mathbb{C})$, and $\Lambda \in(0,+\infty)^{N}$. Assume that $\Lambda$ has rationally independent components. Then $\Sigma(A, B, \Lambda)$ is relatively controllable in some time $T>0$ if and only if

$$
\operatorname{Span}\left\{\left.\Xi_{\mathbf{n}} B e_{j}\left|\mathbf{n} \in \mathbb{N}^{N},\right| \mathbf{n}\right|_{1} \leq d-1, j \in \llbracket 1, m \rrbracket\right\}=\mathbb{C}^{d}
$$

\section{References}

[1] C. E. Avellar and J. K. Hale. On the zeros of exponential polynomials. J. Math. Anal. Appl., 73(2):434-452, 1980.

[2] K. Balachandran, S. Divya, L. Rodríguez-Germá, and J. J. Trujillo. Relative controllability of nonlinear neutral fractional integro-differential systems with distributed delays in control. Math. Methods Appl. Sci., 39(2):214-224, 2016.

[3] Y. Chitour, G. Mazanti, and M. Sigalotti. Persistently damped transport on a network of circles. Trans. Amer. Math. Soc., 2016. Electronically published on October 12, 2016. To appear in print.

[4] Y. Chitour, G. Mazanti, and M. Sigalotti. Stability of non-autonomous difference equations with applications to transport and wave propagation on networks. Netw. Heterog. Media, 11(4):563601, 2016.

[5] D. H. Chyung. On the controllability of linear systems with delay in control. IEEE Trans. Automatic Control, AC-15:255-257, 1970.

[6] K. L. Cooke and D. W. Krumme. Differential-difference equations and nonlinear initialboundary value problems for linear hyperbolic partial differential equations. J. Math. Anal. Appl., 24:372-387, 1968.

[7] J.-M. Coron and H.-M. Nguyen. Dissipative boundary conditions for nonlinear 1-D hyperbolic systems: sharp conditions through an approach via time-delay systems. SIAM J. Math. Anal., 47(3):2220-2240, 2015.

[8] M. Cruz, A. and J. K. Hale. Stability of functional differential equations of neutral type. J. Differential Equations, 7:334-355, 1970.

[9] R. Datko. Linear autonomous neutral differential equations in a Banach space. J. Diff. Equations, 25(2):258-274, 1977.

[10] C. E. de Avellar and S. A. S. Marconato. Difference equations with delays depending on time. Bol. Soc. Brasil. Mat. (N.S.), 21(1):51-58, 1990. 
[11] J. Diblík, M. Fečkan, and M. Pospíšil. On the new control functions for linear discrete delay systems. SIAM J. Control Optim., 52(3):1745-1760, 2014.

[12] J. Diblík and D. Y. Khusainov. Representation of solutions of linear discrete systems with constant coefficients and pure delay. Adv. Difference Equ., pages Art. ID 80825, 13, 2006.

[13] J. Diblík, D. Y. Khusainov, and M. Růžičková. Controllability of linear discrete systems with constant coefficients and pure delay. SIAM J. Control Optim., 47(3):1140-1149, 2008.

[14] E. Fridman, S. Mondié, and B. Saldivar. Bounds on the response of a drilling pipe model. IMA J. Math. Control Inform., 27(4):513-526, 2010.

[15] J. K. Hale, E. F. Infante, and F. S. P. Tsen. Stability in linear delay equations. J. Math. Anal. Appl., 105(2):533-555, 1985.

[16] J. K. Hale and S. M. Verduyn Lunel. Introduction to functional differential equations, volume 99 of Applied Mathematical Sciences. Springer-Verlag, New York, 1993.

[17] J. K. Hale and S. M. Verduyn Lunel. Strong stabilization of neutral functional differential equations. IMA J. Math. Control Inform., 19(1-2):5-23, 2002. Special issue on analysis and design of delay and propagation systems.

[18] D. Henry. Linear autonomous neutral functional differential equations. J. Differential Equations, 15:106-128, 1974.

[19] S. Karthikeyan, K. Balachandran, and M. Sathya. Controllability of nonlinear stochastic systems with multiple time-varying delays in control. Int. J. Appl. Math. Comput. Sci., 25(2):207-215, 2015.

[20] J. Klamka. Relative controllability of nonlinear systems with delays in control. Automatica-J. IFAC, 12(6):633-634, 1976.

[21] B. Klöss. The flow approach for waves in networks. Oper. Matrices, 6(1):107-128, 2012.

[22] W. R. Melvin. Stability properties of functional difference equations. J. Math. Anal. Appl., 48:749-763, 1974.

[23] W. Michiels, T. Vyhlídal, P. Zítek, H. Nijmeijer, and D. Henrion. Strong stability of neutral equations with an arbitrary delay dependency structure. SIAM J. Control Optim., 48(2):763786, 2009.

[24] P. H. A. Ngoc and N. D. Huy. Exponential stability of linear delay difference equations with continuous time. Vietnam Journal of Mathematics, 42(2):1-11, 2014.

[25] D. A. O'Connor and T. J. Tarn. On stabilization by state feedback for neutral differentialdifference equations. IEEE Trans. Automat. Control, 28(5):615-618, 1983.

[26] D. A. O'Connor and T. J. Tarn. On the function space controllability of linear neutral systems. SIAM J. Control Optim., 21(2):306-329, 1983.

[27] A. W. Olbrot. On controllability of linear systems with time delays in control. IEEE Trans. Automatic Control, AC-17(5):664-666, 1972.

[28] L. Pandolfi. Stabilization of neutral functional differential equations. J. Optimization Theory Appl., 20(2):191-204, 1976. 
[29] M. Pospíšil, J. Diblík, and M. Fečkan. On relative controllability of delayed difference equations with multiple control functions. In Proceedings of the International Conference on Numerical Analysis and Applied Mathematics 2014 (ICNAAM-2014), volume 1648, page 130001. AIP Publishing, 2015.

[30] D. Salamon. Control and observation of neutral systems, volume 91 of Research Notes in Mathematics. Pitman (Advanced Publishing Program), Boston, MA, 1984.

[31] R. A. Silkowski. Star-shaped regions of stability in hereditary systems. PhD thesis, Brown University, 1976.

[32] M. Slemrod. Nonexistence of oscillations in a nonlinear distributed network. J. Math. Anal. Appl., 36:22-40, 1971.

[33] E. D. Sontag. Mathematical control theory: Deterministic finite-dimensional systems, volume 6 of Texts in Applied Mathematics. Springer-Verlag, New York, 2 edition, 1998. 